\title{
Damage Identification by the Kullback-Leibler Divergence and Hybrid Damage Index
}

\author{
Shaohua Tian, Zhibo Yang, Zhengjia He, and Xuefeng Chen \\ State Key Laboratory for Manufacturing Systems Engineering, Xian Jiaotong University, Xi'an 710049, China \\ Correspondence should be addressed to Zhengjia He; hzj@mail.xjtu.edu.cn
}

Received 4 June 2013; Accepted 19 September 2013; Published 17 March 2014

Academic Editor: Gyuhae Park

Copyright ( 2014 Shaohua Tian et al. This is an open access article distributed under the Creative Commons Attribution License, which permits unrestricted use, distribution, and reproduction in any medium, provided the original work is properly cited.

\begin{abstract}
The hybrid damage index (HDI) is presented as a mean for the damage identification in this paper, which is on the basis of the Kullback-Leibler divergence (KLD) and its approximations. The proposed method is suitable for detecting damage in onedimensional structure and delamination in laminated composite. The first step of analysis includes obtaining the mode data of the structure before and after the damage, and then the KLD and its approximations are obtained. In addition, the HDI is obtained on the basis of the KLD and its approximations, utilizing the natural frequencies and mode shape at the same time. Furthermore, the modal strain energy (MSE) method is employed to verify the efficiency of the proposed method. Finally, to demonstrate the capability of the proposed method, examples of the beam and laminated composite are applied for checking the present approaches numerically, and the final results validate the effective and accurate performance of the present technique.
\end{abstract}

\section{Introduction}

As evidenced by the vast literature in the damage detection, the structural health monitoring has become an increasingly crucial issue. To data, significant efforts have been made by researchers in the damage identification. The presence of damage generally produces changes in the structural stiffness matrix. Meanwhile, these changes are accompanied with changes in the structural modal parameters. This phenomenon has been widely noted and used by researchers in distinguishing the damage. However, using different modal parameters correlated with other relevant information in the damage identification may get very various results with varying accuracy. For this reason, seeking a proper selection or combination of dynamic parameters is an imperative purpose.

From the perspective of the damage detection, Park et al. [1] reviewed the piezoelectric impedance-based structural health monitoring and applied it in the damage detection of civil structural components [2]. Sekhar [3] provided an excellent review on research advances in damage detection areas over the twenty years. Fan and Qiao [4] reviewed vibrationbased damage identification methods and gave a comparative study on the damage detection, and the strain-based damage index for the structural damage identification was reviewed by $\mathrm{Li}$ [5]; the recurrence quantification analysis has emerged as a useful tool for detecting subtle nonstationarities and changes in the time-series data; Nichols et al. [6] extended the recurrence quantification analysis method to multivariate observations for the damage detection. Sun et al. [7] applied the manifold subspace distance to assess the damage. Yan and Golinval [8] utilized the singular value decomposition to obtain characteristic subspaces; then the statistical process technique is adopted for the damage detection of the aircraft wing. Bueno and Sinou [9] employed the Gramian matrices to detect damage in a cantilever beam. Furthermore, wavelet $[10,11]$ and dimension $[12]$ are also used to detect damage; Liu et al. [13] employed the frequency slice wavelet transform to accomplish the damage detection.

Laminated composite have been widely applied to aeronautic structures as well as automobile and wind power equipment; the reason for that is because laminated composite possesses many merits, such as lighter weight, higher stiffness, heat insulation and preservation, and antiradiation. Usually, this kind of the structural material is made of very 
thin carbon fiber, glass fiber, and so on. One of the advantages over the tradition material is the light weight, typically only $10-15 \%$ of that of a steel structure in condition of obtaining the same stiffness and strength.

Under repeated or impact loads in the serving process, these laminated composites are subjected to various forms of the damage as a result of manufacturing defects or in-service factors, mostly the delamination. Such delamination presents a serious threat to the proper performance of the laminated composite and becomes an obstacle to more extensive usage of the laminated composite. Thus the monitoring of the hidden delamination in the laminated composite is critical in the engineering practice. Meanwhile, the use of vibrationbased techniques as nondestructive testing methods for the damage monitoring of the laminated composite is a field attracting the interest of many researchers.

Up to now, remarkable efforts have been made by researchers in the identification of delamination; the presence of delamination generally produces changes in the structural physical properties. Likewise, these changes are accompanied with changes in the modal parameters of the structure. This phenomenon has been widely noted and used by researchers for distinguishing the delamination. However, using different modal parameters correlated with other relevant information in delamination may get very various results. For this reason, seeking a proper selection or combination of dynamic parameters is a crucial thing.

With respect to detecting the delamination in the composited composite, Nalawade et al. [14] utilized the embedded Hi-Bi photonic crystal fiber to detect the delamination in the laminated composite. Wang et al. [15, 16] employed the Lamb to identify the damage in composite. Yang et al. [17] applied the inner product vector for the vibration response of the laminated composite before and after the presence of the delamination to identify the delamination successfully. Based on the continuum damage mechanics, Shang et al. [18] employed the subset selection technique to complete the delamination identification of laminated composite plates. Based on the novel Laplacian scheme and multiresolution modal curvatures; Kim et al. [19] applied the smooth transition displacement to construct the damage index in the delamination identification. In addition, Liu et al. accomplished the delamination identification by the condition recognition [20] and time-frequency approach [21], respectively.

Worden and Burrows [22] adopted the stiffness degradation method to simulate the damage in aluminum sandwich beams; damage identification results by neural network show that the curvature calculated by the first displacement mode is more sensitive to the damage. Xu et al. and Liu et al. [23] calculated anisotropic laminated plate mode parameters by simulating the delamination through embedding bar units into two layers and the edge-based smoothed point interpolation method [24]; then the delamination in the anisotropic laminated plate is identified by the neural network. Valoor and Chandrashekhara [25] established the delamination composited beam model by the first-order shear deformation theory; then they calculated the dynamic response of the composited beam in different boundary conditions; mode parameters were employed as the input sample of the neural network; the identification result shows that the displacement mode has a relative higher accuracy, and Katz [26] proposed the conception of the fractal dimension. In the process of calculating the fractal dimension, a moving window was employed; firstly, the fractal dimension in the moving windows is calculated; secondly, every fractal dimension of moving windows was calculated when the moving window advances along the beam. Through the location of the peak of the fractal dimension, the delamination is identified. While Wang and Deng [27] applied the Haar wavelet to process the continuous digital signal to detect the damage, Wu and Law [28] made use of the uniform load surface and detected the damage in an aluminum plate. Then they [29] proposed an anisotropic damage model in the thin plate and identified the damage by the uniform load surface curvature and furthermore predicted the length of the crack on the basis of conservation law of potential energy. In recent years, they used the covariance of the response and the measurement noise [30] to detect damage. Lu et al. [31] employed Lamb wave signals as the input sample of the artificial neural network to identify the crack in aluminum plate. Radzieński et al. [32] proposed an improved method for the damage detection in beam with the basis of experimental modal parameters of a clamped beam, whereas the identification of the two-dimensional structure is not studied.

The KLD is a widely employed tool in the statistics and pattern recognition [33], which is known as the relative entropy; Eguchi and Copas [34] interpreted the KLD with the Neyman-Pearson lemma and overviewed the KLD in the information geometry. Smith et al. [35] employed the KLD between true and candidate models to select the number of states and variables simultaneously in Markov-switching regression models, whereas the KLD is not symmetrical and it adapts to the triangle inequality. To overcome these shortcomings, the $J$ divergence (JD) and the $\chi^{2}$ statistic distribution (SD) are adopted. Furthermore, the JD is the symmetrized version of the KLD and the $\chi^{2}$ statistic distribution is an approximation to the KLD from the statistic point of view; moreover, the JD is an usual tool for measuring the similarity between probability density functions. Mathiassen et al. [36] employed the KLD, the $\chi^{2}$ statistic distribution, and the JD to measure the texture similarity. Then Puzicha et al. [37] also utilized them to evaluate the dissimilarity for the color empirical.

In this study, the KLD and its approximation are applied to identify the damage in the beam and the delamination in the laminated composite. In order to obtain more accurate results of detecting the damage and delamination, especially the multiple damage and delamination, an improving method named the hybrid damage index is adopted. The one-dimensional beam is employed to validate the work of Radzieński et al. [32], firstly, and then the twodimensional laminated composite is applied to extend the object of the method, which is not included in the work of Radzieński et al.; furthermore, the method of the modal strain energy is also employed to verify the efficiency of the proposed method. Meanwhile, the mode strain energy 
is employed to verify the efficiency of the proposed method. Finally, numerical results validate the reliability and accuracy of the proposed method.

\section{Algorithms}

2.1. KLD. KLD is a fundamental concept about the expected log-likelihood ratio in statistics, which is a widely used tool in statistics and pattern recognition.

Assuming $P(x)$ and $Q(x)$ to be two probability distributions with probability density functions $P(x)$ and $q(x)$, respectively, the KLD from the $P(x)$ to the $Q(X)$ is defined as [33]

$$
D(P, Q)=\int p(x) \log \frac{p(x)}{q(x)} d x,
$$

where $E_{P}$ denotes taking the expectation operator over the distribution $P$.

Considering a vibration problem of a beam, the $p(x)$ and $q(x)$ can be replaced by the $l$ th mode shapes $\phi^{l}(x)$ and $\phi^{d l}(x)$ of the intact and damaged beam; thus the corresponding KLD of the beam is given as follows:

$$
\mathrm{KLD}^{l}=\int_{0}^{L} \phi^{d l}(x) \log \frac{\phi^{d l}(x)}{\phi^{l}(x)} d x .
$$

Supposing that the beam structure is divided into $n$ elements, the KLD of the $i$ th element is defined as follows:

$$
\operatorname{KLD}_{i}^{l}=\int_{a_{i}}^{a_{i+1}} \phi_{i}^{d l}(x) \log \frac{\phi_{i}^{d l}(x)}{\phi_{i}^{l}(x)} d x,
$$

where $a_{i}$ and $a_{i+1}$ are the location of the $i$ th and $(i+1)$ th node.

To account for all available modes, the KLD along the beam employed for the damage detection can be expressed as

$$
\mathrm{KLD}_{i}=\sum_{l=1}^{N M} \int_{a_{i}}^{a_{i+1}} \phi_{i}^{d l}(x) \log \frac{\phi_{i}^{d l}(x)}{\phi_{i}^{l}(x)} d x
$$

By the same token, the KLD of the $(i, j)$ th point in the laminated composite is given as

$$
\mathrm{KLD}_{i, j}=\sum_{l=1}^{N M} \int_{b_{j}}^{b_{j+1}} \int_{a_{i}}^{a_{i+1}} \phi_{i, j}^{d l}(x) \log \frac{\phi_{i, j}^{d l}(x)}{\phi_{i, j}^{l}(x)} d x d y .
$$

2.2. JD. JD [38] is employed to measure the distance between two probabilistic distributions or the similarity of two vectors, which also can be applied to establish and recognize the sample of the working status. Furthermore, the JD is given as

$$
J\left(F_{1}, F_{2}\right)=\frac{1}{2 N} \sum_{i=1}^{N}\left(\frac{f_{1 i}}{f_{2 i}}+\frac{f_{2 i}}{f_{1 i}}\right)-1,
$$

in which $f_{1 i}$ and $f_{2 i}$ are elements of sets $F_{1}$ and $F_{2}$ of the sample and $N$ is the number of the sample set.

The JD of the $i$ th element in a beam is obtained by the same token as the KLD and is expressed as

$$
\mathrm{JD}_{i}^{l}\left(\phi_{i}^{l}, \phi_{i}^{d l}\right)=\frac{1}{2}\left(\frac{\phi_{i}^{l}}{\phi_{i}^{d l}}+\frac{\phi_{i}^{d l}}{\phi_{i}^{l}}\right)-1 .
$$

To account for all available modes, the JD along the beam employed for the damage detection can be expressed as

$$
\mathrm{JD}_{i}=\sum_{l=1}^{N M}\left[\frac{1}{2}\left(\frac{\phi_{i}^{l}}{\phi_{i}^{d l}}+\frac{\phi_{i}^{d l}}{\phi_{i}^{l}}\right)-1\right] .
$$

Similarly, the JD of the $(i, j)$ point in the laminated composite is expressed as

$$
\mathrm{JD}_{i, j}=\sum_{l=1}^{N M}\left[\frac{1}{2}\left(\frac{\phi_{i, j}^{l}}{\phi_{i, j}^{d l}}+\frac{\phi_{i, j}^{d l}}{\phi_{i, j}^{l}}\right)-1\right] .
$$

2.3. $\chi^{2}$ Statistic Distribution. $\chi^{2}$ statistic distribution [36] is an approximation to the KLD from the statistic point of view, and it is defined as

$$
D_{\chi^{2}}(q, p)=\sum_{j=1}^{N} \sum_{l=1}^{L-1} \frac{\left[q_{j}(l)-q_{j}(l)\right]^{2}}{q_{j}(l)+q_{j}(l)} .
$$

The $\chi^{2}$ statistic distribution of the $l$ th mode is given as

$$
\mathrm{SD}^{l}=\frac{\left(\phi^{d l}-\phi^{l}\right)^{2}}{\phi^{d l}+\phi^{l}} \text {. }
$$

The $\chi^{2}$ statistic distribution of the $i$ th element is expressed as

$$
\mathrm{SD}_{i}^{l}=\frac{\left(\phi_{i}^{d l}-\phi_{i}^{l}\right)^{2}}{\phi_{i}^{d l}+\phi_{i}^{l}} .
$$

To account for all the available modes, the $\chi^{2}$ distribution along the beam employed for the damage detection can be expressed as

$$
\mathrm{SD}_{i}=\sum_{l=1}^{N M} \frac{\left(\phi_{i}^{d l}-\phi_{i}^{l}\right)^{2}}{\phi_{i}^{d l}+\phi_{i}^{l}}
$$

Similarly, the $\chi^{2}$ distribution of the $(i, j)$ th point in the laminated composite is given as

$$
\mathrm{SD}_{i, j}=\sum_{l=1}^{N M} \frac{\left(\phi_{i, j}^{d l}-\phi_{i, j}^{l}\right)^{2}}{\phi_{i, j}^{d l}+\phi_{i, j}^{l}} .
$$

2.4. Modal Strain Energy of the One-Dimensional Beam. The strain energy $U$ of a Bernoulli-Euler beam [39] is calculated as follows:

$$
U=\frac{1}{2} \int_{0}^{L} E I\left(\frac{\partial^{2} w}{\partial^{2} x}\right) d x
$$


where EI represents the bending rigidity (stiffness) of the beam and $L$ denotes the length of the beam; meanwhile $w$ indicates the transverse displacement of the beam.

Considering a vibration problem of a one-side clamped beam which is subdivided into $N$ subregions, the location of each subregion is able to be represented by $\left(a_{i}, a_{i+1}\right)$, and the corresponding strain energy associated with that subregion for $l$ th mode $\phi^{l}(x)$ can be expressed as

$$
U_{i}^{l}=\frac{1}{2} \int_{a_{i}}^{a_{i+1}}(E I)_{j}\left(\frac{\partial^{2} \phi^{l}(x)}{\partial^{2} x}\right) d x .
$$

Hence the fractional energy of that subregion is

$$
F_{i}^{l}=\frac{U_{i}^{l}}{U^{l}}, \quad \sum_{j=1}^{N_{m}} F_{i}^{l}=1 .
$$

In the same way, the corresponding fractional energy of that subregion for a damaged beam is given as

$$
\begin{gathered}
U_{i}^{d l}=\frac{1}{2} \int_{a_{i}}^{a_{i+1}}(E I)_{d i}\left(\frac{\partial^{2} \phi^{d l}(x)}{\partial^{2} x}\right) d x, \\
F_{i}^{d l}=\frac{U_{i}^{d l}}{U^{d l}}, \quad \sum_{j=1}^{N_{m}} F_{i}^{d l}=1 .
\end{gathered}
$$

As a consequence, the damage index for each subregion along the beam is given by

$$
\beta_{i}=\frac{\sum_{l=1}^{N} F_{i}^{d l}}{\sum_{l=1}^{N} F_{i}^{l}} .
$$

A normalized damage index can be expressed as

$$
Z_{i}=\frac{\beta_{i}-\bar{\beta}_{i}}{\sigma_{\beta_{i}}}
$$

in which $\bar{\beta}_{i}$ represents the mean of $\beta_{i}$ and $\sigma_{\beta_{i}}$ denotes the standard deviation of $\beta_{i}$.

2.5. Modal Strain Energy of the Laminated Composite. For an orthotropic laminated composite with a dimension of $a \times b$, the total strain energy $U[40]$ is given by

$$
\begin{aligned}
U=\frac{1}{2} \int_{0}^{b} \int_{0}^{a}[ & D_{11}\left(\frac{\partial^{2} w}{\partial x^{2}}\right)^{2} \\
& +D_{22}\left(\frac{\partial^{2} w}{\partial y^{2}}\right)^{2}+2 D_{12} \frac{\partial^{2} w}{\partial x^{2}} \frac{\partial^{2} w}{\partial y^{2}} \\
& +4\left(D_{16} \frac{\partial^{2} w}{\partial x^{2}}+D_{26} \frac{\partial^{2} w}{\partial y^{2}}\right) \frac{\partial^{2} w}{\partial x \partial y} \\
& \left.+4 D_{66}\left(\frac{\partial^{2} w}{\partial x \partial y}\right)^{2}\right] d x d y,
\end{aligned}
$$

where $D_{i j}$ denotes the bending stiffness of the laminated composite; $w$ indicates the deflection of the laminated composite; $\partial^{2} w / \partial x^{2}$ and $\partial^{2} w / \partial y^{2}$ are the bending curvature of the laminated composite; $\partial^{2} w / \partial x \partial y$ is the twisting curvature of the laminated composite.

Supposing that the laminated composite structure is subdivided into $N_{x} \times N_{y}$ subregions and the location of each subregion is represented by $\left(x_{i}, x_{i+1}\right)$ and $\left(y_{i}, y_{i+1}\right)$, then the corresponding strain energy associated with the $(i, j)$ subregion for the $l$ th mode shape $\phi^{l}(i, j)$ is given as follows:

$$
\begin{aligned}
& U_{i, j}^{l} \\
& =\frac{1}{2} \int_{y_{j}}^{y_{j+1}} \int_{x_{i}}^{x_{i+1}}\left[D_{11}\left(\frac{\partial^{2} \phi^{l}(x, y)}{\partial x^{2}}\right)^{2}\right. \\
& +D_{22}\left(\frac{\partial^{2} \phi^{l}(x, y)}{\partial y^{2}}\right)^{2} \\
& +2 D_{12} \frac{\partial^{2} \phi^{l}(x, y)}{\partial x^{2}} \frac{\partial^{2} \phi^{l}(x, y)}{\partial y^{2}} \\
& +4\left(D_{16} \frac{\partial^{2} \phi^{l}(x, y)}{\partial x^{2}}+D_{26} \frac{\partial^{2} \phi^{l}(x, y)}{\partial y^{2}}\right) \\
& \times \frac{\partial^{2} \phi^{l}(x, y)}{\partial x \partial y} \\
& \left.+4 D_{66}\left(\frac{\partial^{2} \phi^{l}(x, y)}{\partial x \partial y}\right)^{2}\right] d x d y .
\end{aligned}
$$

Thus the fractional energy of that subregion is

$$
\begin{gathered}
F_{i, j}^{l}=\frac{U_{i, j}^{l}}{U^{l}}, \\
\sum_{j=1}^{N_{y}} \sum_{i=1}^{N_{x}} F_{i, j}^{l}=1 .
\end{gathered}
$$

For the laminated composite with delamination, some analogous equations can be defined using the damaged mode shape, which are given by

$$
\begin{aligned}
& U^{d l} \\
& =\frac{1}{2} \int_{0}^{b} \int_{0}^{a}\left[D_{11}\left(\frac{\partial^{2} \phi^{d l}(x, y)}{\partial x^{2}}\right)^{2}+D_{22}\left(\frac{\partial^{2} \phi^{d l}(x, y)}{\partial y^{2}}\right)^{2}\right. \\
& +2 D_{12} \frac{\partial^{2} \phi^{d l}(x, y)}{\partial x^{2}} \frac{\partial^{2} \phi^{d l}(x, y)}{\partial y^{2}} \\
& +4\left(D_{16} \frac{\partial^{2} \phi^{d l}(x, y)}{\partial x^{2}}+D_{26} \frac{\partial^{2} \phi^{d l}(x, y)}{\partial y^{2}}\right) \\
& \left.\times \frac{\partial^{2} \phi^{d l}(x, y)}{\partial x \partial y}+4 D_{66}\left(\frac{\partial^{2} \phi^{d l}(x, y)}{\partial x \partial y}\right)^{2}\right] d x d y,
\end{aligned}
$$




$$
\begin{aligned}
& U_{i, j}^{d l} \frac{1}{2} \int_{y_{j}}^{y_{j+1}} \int_{x_{i}}^{x_{i+1}}\left[D_{11}\left(\frac{\partial^{2} \phi^{d l}(x, y)}{\partial x^{2}}\right)^{2}+D_{22}\left(\frac{\partial^{2} \phi^{d l}(x, y)}{\partial y^{2}}\right)^{2}\right. \\
&+2 D_{12} \frac{\partial^{2} \phi^{d l}(x, y)}{\partial x^{2}} \frac{\partial^{2} \phi^{d l}(x, y)}{\partial y^{2}} \\
&+4\left(D_{16} \frac{\partial^{2} \phi^{d l}(x, y)}{\partial x^{2}}+D_{26} \frac{\partial^{2} \phi^{d l}(x, y)}{\partial y^{2}}\right) \\
& \times \frac{\partial^{2} \phi^{d l}(x, y)}{\partial x \partial y} \\
&\left.+4 D_{66}\left(\frac{\partial^{2} \phi^{d l}(x, y)}{\partial x \partial y}\right)^{2}\right] d x d y \\
& \sum_{j=1}^{N_{y}} \sum_{i=1}^{N_{x}} F_{i, j}^{d l}=1 . \\
& F_{i, j}^{d l}=\frac{U_{i, j}^{d l}}{U^{d l}}
\end{aligned}
$$

To account for all the available modes, the delamination index for each subregion along the laminated composite is given by

$$
\beta_{i, j}=\frac{\sum_{l=1}^{N_{m}} F_{i, j}^{d l}}{\sum_{l=1}^{N_{m}} F_{i, j}^{l}}
$$

A normalized damage index is given by

$$
Z_{i, j}=\frac{\beta_{i j}-\bar{\beta}_{i j}}{\sigma_{\beta_{i j}}}
$$

in which $\bar{\beta}_{i, j}$ represents the mean of $\beta_{i, j}, \sigma_{\beta i, j}$ denotes the standard deviation of $\beta_{i, j}$.

\subsection{The Hybrid Damage Index}

2.6.1. The One-Dimensional Hybrid Damage Index. The process of calculating the one-dimensional hybrid damage index is given as follows.

(1) Calculate the change of natural frequencies of the beam before and after the occurrence of the damage as follows:

$$
\Delta \omega_{l}=\omega_{l}-\omega_{l}^{d}
$$

(2) Certification of the following inequality is done: provided $\max \left(\Delta \omega_{l}\right)-\min \left(\Delta \omega_{l}\right)<2 \delta$, in which $\delta$ indicates the threshold; thereby it is assumed that the damage is too small to be detected.

(3) In order to improve the identification precision and reduce the boundary effect, the spline extrapolation of the "measured" point for the KLD with the number of the size of the filtering window is done.

(4) Reconstructing the KLD of the beam before and after the occurrence of the damage with the weight coefficient $a_{i}$ is as follows:

$$
\widetilde{D}_{i}^{l}=\sum_{i=1}^{i} a_{i} D_{i}^{l}
$$

in which $D_{i}^{l}$ indicates the KLD based on the $l$ th mode shape of the $i$ th point of the intact and damaged beam and $a_{i}$ denotes the weighting coefficient with the value of $1 / k$ for the $k$ th subregion of the beam structure.

(5) If the number of obtained points is not enough, the cubic extrapolation is adopted to increase the "measured" points with adequate number of points.

(6) Calculate the curvature of the reconstructed $\widetilde{D}_{i}^{l}$. The curvature $v_{i}^{l}$ for the $l$ th $\widetilde{D}_{i}^{l}$ with respect to the $i$ th subregion is given by

$$
v_{i}^{l}=\frac{\widetilde{D}_{i-1}^{l}+\widetilde{D}_{i+1}^{l}-2 \widetilde{D}_{i}^{l}}{h^{2}},
$$

in which $h$ represents the length between two adjoining points.

(7) Normalize the curvature $v_{i}^{l}$ dimensionless to $[0,1]$ range

$$
\widetilde{v}_{i}^{l}=\frac{v_{i}^{l}-\min \left(v_{i}^{l}\right)}{\max \left(v_{i}^{l}\right)-\min \left(v_{i}^{l}\right)}
$$

(8) Construct the function $R$ for the damage identification.

The function $R$ of the damage identification for the $l$ th mode shape with respect to the $i$ subregion is defined to be

$$
R_{i}^{l}=1-\left|\left(\widetilde{v}_{i}^{l}\right)^{2}-\Delta \omega_{l}\right|
$$

(9) Normalize the curvature $R_{i}^{l}$ dimensionless to $[0,1]$ range

$$
\widetilde{R}_{i}^{l}=\frac{R_{i}^{l}-\min \left(R_{i}^{l}\right)}{\max \left(R_{i}^{l}\right)-\min \left(R_{i}^{l}\right)} .
$$

(10) To account for all available modes, a hybrid damage index $\mathrm{KLDHDI}_{i}$ based on the KLD for the $l$ th mode with respect to the $i$ subregion based on the KLD is given as follows:

$$
\mathrm{KLDHDI}_{i}=\widetilde{R}_{i}^{l} \frac{\left(\sum_{i=1}^{N_{m}}\left[\left(v_{i}^{d l}\right)^{2}+\sum_{i=1}^{N}\left(v_{i}^{d l}\right)^{2}\right] \sum_{i=1}^{N}\left(v_{i}^{l}\right)^{2}\right)}{\sum_{i=1}^{N_{m}}\left[\left(v_{i}^{l}\right)^{2}+\sum_{i=1}^{N}\left(v_{i}^{l}\right)^{2}\right] \sum_{i=1}^{N}\left(v_{i}^{d l}\right)^{2}} \text {. }
$$




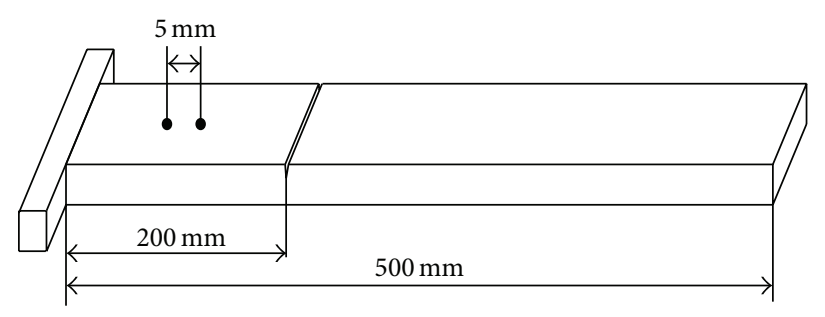

(a) Damage scenario of the single kind of damage

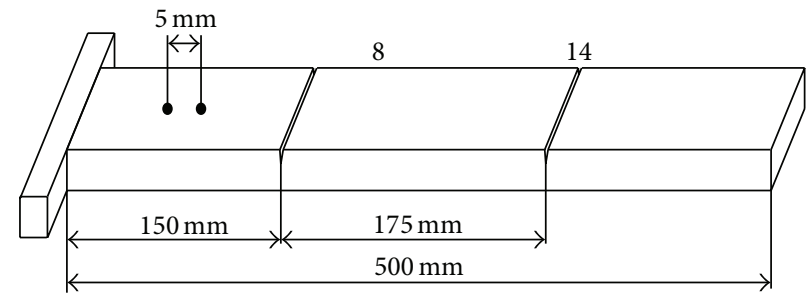

(b) Damage scenario of two kinds of damage

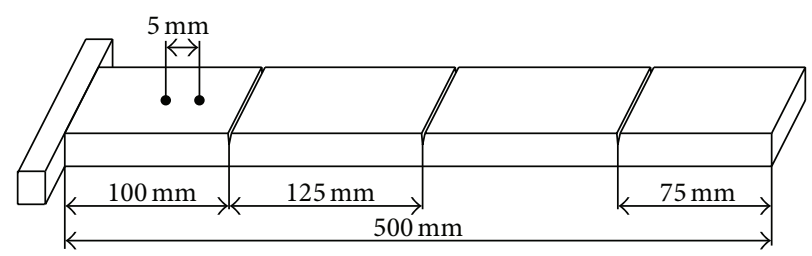

(c) Damage scenario of two kinds of damage

FIgURE 1: Damage scenarios of the beam.

2.6.2. The Two-Dimensional Hybrid Damage Index. For the laminated composite, the similar hybrid delamination index $\mathrm{KLDHDI}_{i, j}$ is obtained in the same way and is given as

$$
\mathrm{KLDHDI}_{i, j}=\widetilde{R}_{i, j}^{l} \frac{\left(\sum_{j=1}^{N_{m}}\left[\left(v_{i, j}^{d l}\right)^{2}+\sum_{i=1}^{N}\left(v_{i, j}^{d l}\right)^{2}\right] \sum_{i=1}^{N}\left(v_{i, j}^{l}\right)^{2}\right)}{\sum_{j=1}^{N_{m}}\left[\left(v_{i, j}^{l}\right)^{2}+\sum_{i=1}^{N}\left(v_{i, j}^{l}\right)^{2}\right] \sum_{i=1}^{N}\left(v_{i, j}^{d l}\right)^{2}},
$$

in which the value of the weight coefficient $a_{i, j}$ is defined as $1 /(i \times j)$ for the subregion $(i, j)$ in the laminated composite.

Finally the similar hybrid damage index based on the $J$ divergence (JDHDI) and $\chi^{2}$ statistical distribution (SDHDI) can be obtained by the same token.

\section{The Verification of the Proposed Method by Numerical Results}

In previous preceding sections it has been shown how to utilize the proposed method in order to assess the location of the damage and delamination. Aiming to account for the practical use of results above with the analysis of real cases, following sections are devoted to outlining some application of numerical simulation samples.

To simulate the real experiment environment, we employed the first three modes of the structure before and after the presence of the damage and delamination in the certification of the proposed method by numerical results.

3.1. Case Study I: Beam. In this section, an isotropic beam is employed to elucidate the performance of the proposed method.

3.1.1. Material Properties. To certify the proposed method, a one-side clamped beam is considered. Material properties are taken from steel where the elastic modulus $E=2.06 \times$
$10^{11} \mathrm{~Pa}$, Poisson's ration $\nu=0.3$, and the density $\rho=$ $7800 \mathrm{~kg} / \mathrm{m}^{3}$. The geometrical dimension is given as follows: the length of the beam is $5 \mathrm{~m}$, the area of the cross-section (cross-sectional area) is $0.25 \times 0.2 \mathrm{~m}^{2}$, and the geometrical moment of the inertia for the beam cross-section is $1.67 \times$ $10^{-4} \mathrm{~m}^{4}$.

3.1.2. The Damage Detection of the Beam. The beam is divided into 100 elements, and the single damage is numerically simulated in elements 41 to 45 by reducing the elasticity modulus to the half. Furthermore, in order to detect the multiple damages, two kinds of damage are numerically simulated by reducing the elasticity modulus to the half in elements 31 to 35 and elements 71 to 75 , and the reduction of the elasticity modulus in elements 21 to 25 , elements 51 to 55 , and elements 81 to 85 to half is employed to simulate three kinds of damage, which is named as the first damage scenario. In order to test the ability of the proposed method to differentiate extents of the damage, the elasticity modulus was reduced to forty percent to simulate the second damage scenario. Finally, all the damage scenarios are shown in Figure 1.

With the aid of the modal analysis in the FE techniques, the first three modes of the intact and the damaged beam are obtained. Owing to the presence of the damage, the change of the mode shape which cannot be visible is induced. The KLD and its approximations are adopted with the intension of detecting the damage in the beam, and then a hybrid damage index based on the KLD and its approximation is applied with the purpose of improving the reliability and accuracy of the above method; all results of the first damage scenario are shown in Figures 2, 3, and 4. Meanwhile, corresponding results are shown in Figures 5, 6, and 7.

3.1.3. Discussions. The appearance and location of the damage are observed through the abrupt anomaly of the curve. Thereby, the MSE, KLD and its approximations, and HDI 


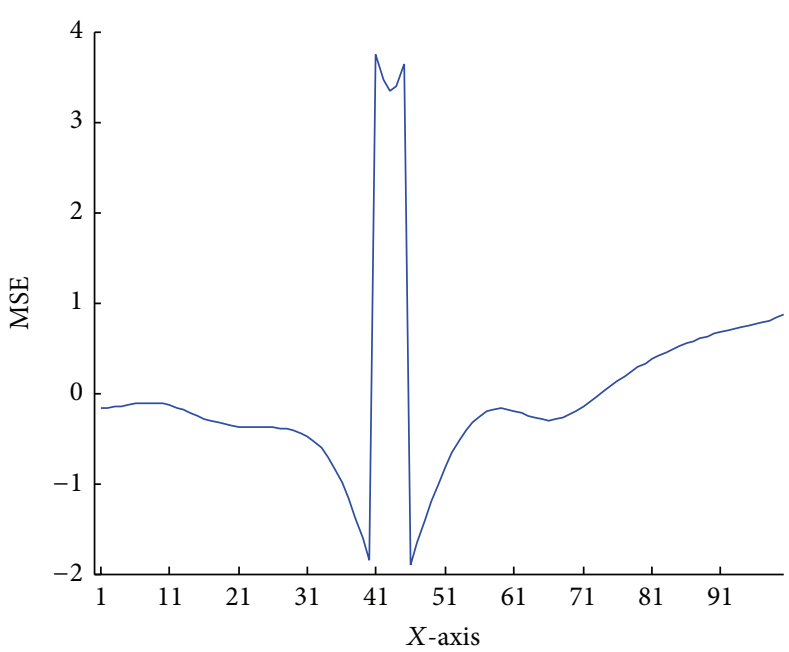

(a) MSE of the single kind of damage

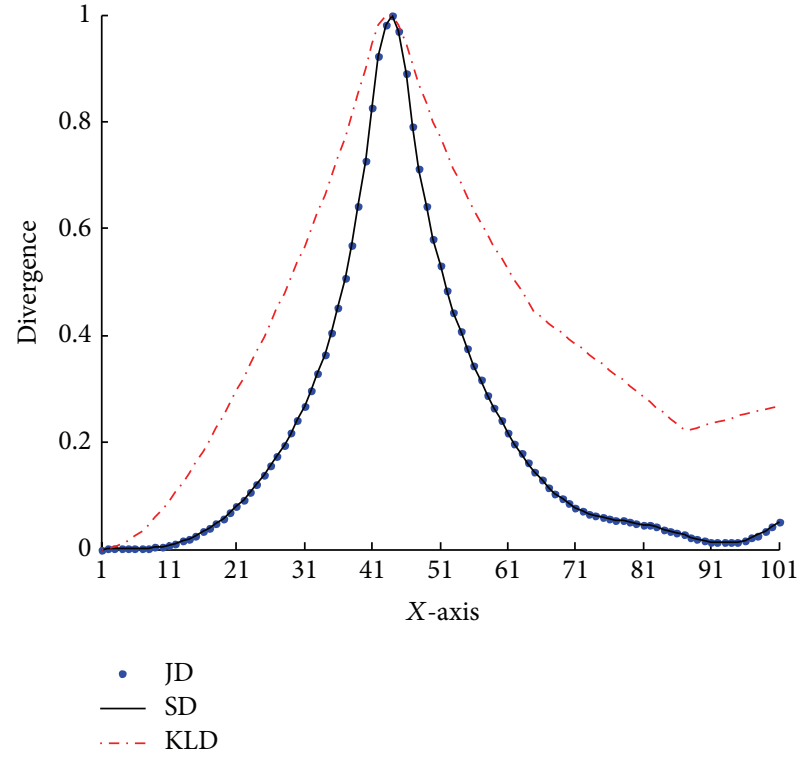

(b) Divergence of the single kind of damage

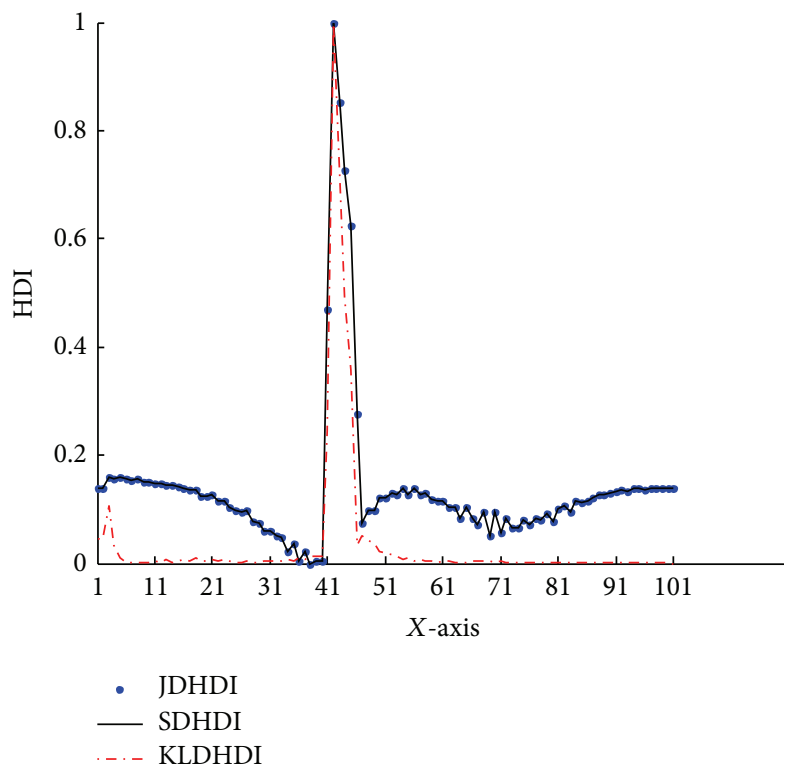

(c) HDI of the single kind of damage

FIGURE 2: Results of the single kind of damage in D1.

based on the KLD and its approximations, respectively, are able to detect the all damage in the first and second damage scenarios in the view of damage identification results from Figures 2 to 7.

However, the value of the KLD is almost larger than JD and $\chi^{2}$ distribution along the beam, and the falling velocity of the JD and $\chi^{2}$ distribution in the vicinity of the damage location is faster than the KLD. As a consequence, the localized ability of the JD and $\chi^{2}$ distribution in the vicinity of the damage location is stronger than the KLD; it demonstrates that the aggregation of the JD and $\chi^{2}$ distribution is better than the KLD, which means that the JD and $\chi^{2}$ distribution are more sensitive to the damage than the KLD. Hence the ability of the JD and $\chi^{2}$ distribution to detect the damage is stronger than the KLD. Furthermore, the JD is almost the same as the $\chi^{2}$ distribution, and it is found that the sensitivity to damage of the JD is equal to the $\chi^{2}$ distribution. So it is able to draw a conclusion that JD and $\chi^{2}$ distribution are capable of overcoming the shortcoming of the KLD.

It is worth noting out that the error of the multidamage identification is larger than the corresponding error of the single damage identification for KLD and its approximations. Furthermore, the value of peaks of the damage on diverse locations varies; thus different locations of the damage can 

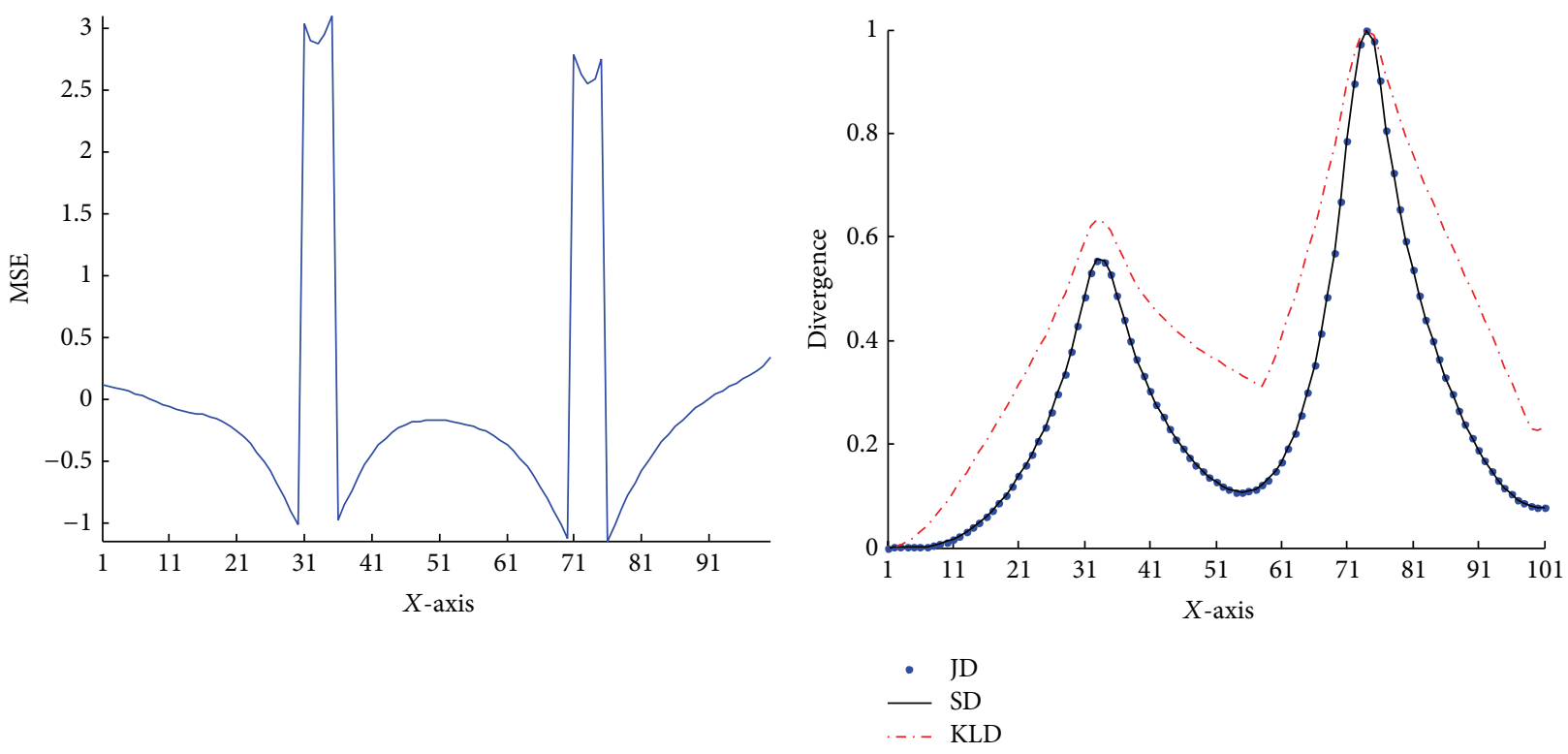

(a) MSE of two kinds of damage

(b) Divergence of two kinds of damage

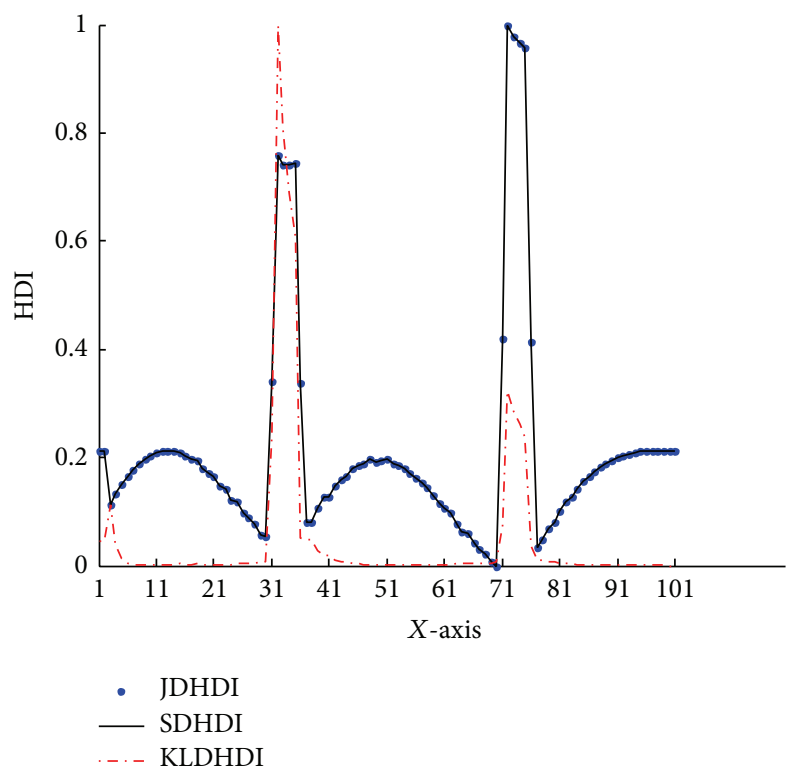

(c) HDI of two kinds of damage

Figure 3: Results of two kinds of damage in D1.

result in various errors of the damage identification. Generally speaking, the error of the damage identification is smaller when the distance between the location of damage and the clamped end is shorter except for the damage identification of three kinds of damage by the JD and $\chi^{2}$ distribution in the second scenario. And the reason for that is that the first mode shape plays an important role in the above phenomenon. Furthermore, the longer the distance between the clamped end and the measured point is, the larger the first mode shape is.

On the basis of the KLD and its approximation, the hybrid damage index is obtained, and it is found that the error of the damage identification by the HDI is much smaller than the corresponding KLD and its approximations. Hence a noticeable improvement of the damage identification by the hybrid damage index in comparison with the KLD is observed, in the multiple damage detection, peaks in locations of damage alter greater in comparison with the corresponding, which is able to affect the accuracy of the damage detection. However the HDI based on the KLD and its approximations is smaller than the corresponding MSE in the undamaged region. The HDI based on the KLD and its approximations is more sensitive to the MSE.

In the KLD and its approximations, the KLD is larger than the JD and $\chi^{2}$ distribution in the undamaged region, whereas 


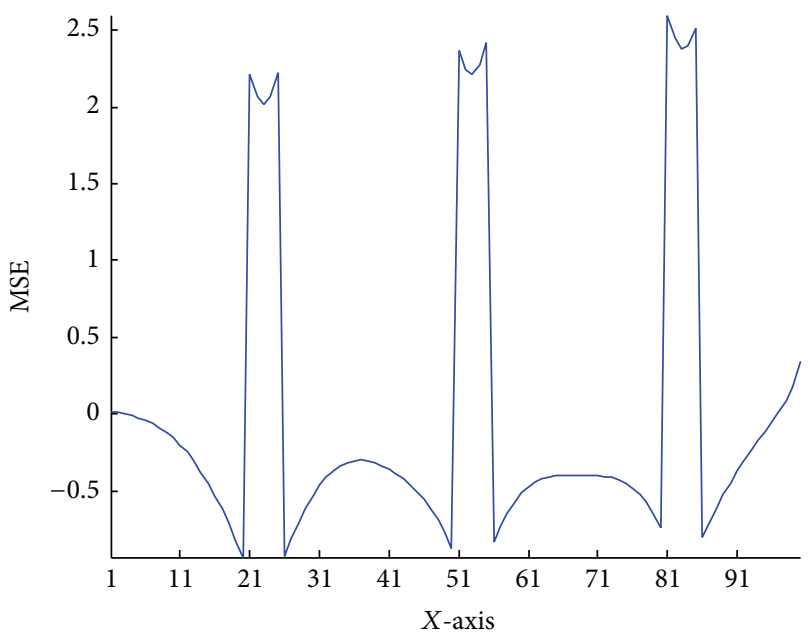

(a) MSE of two kinds of damage

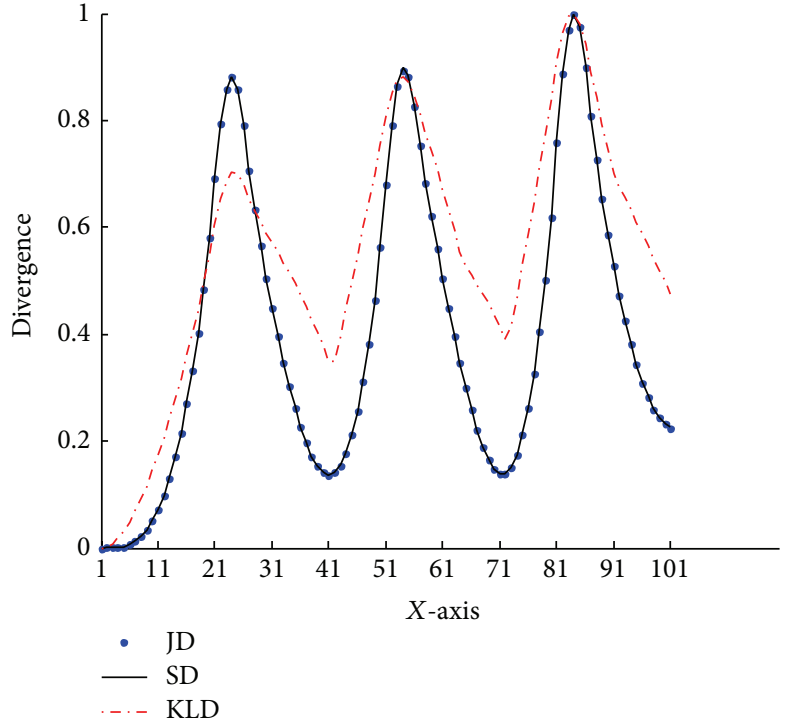

(b) Divergence of three kinds of damage

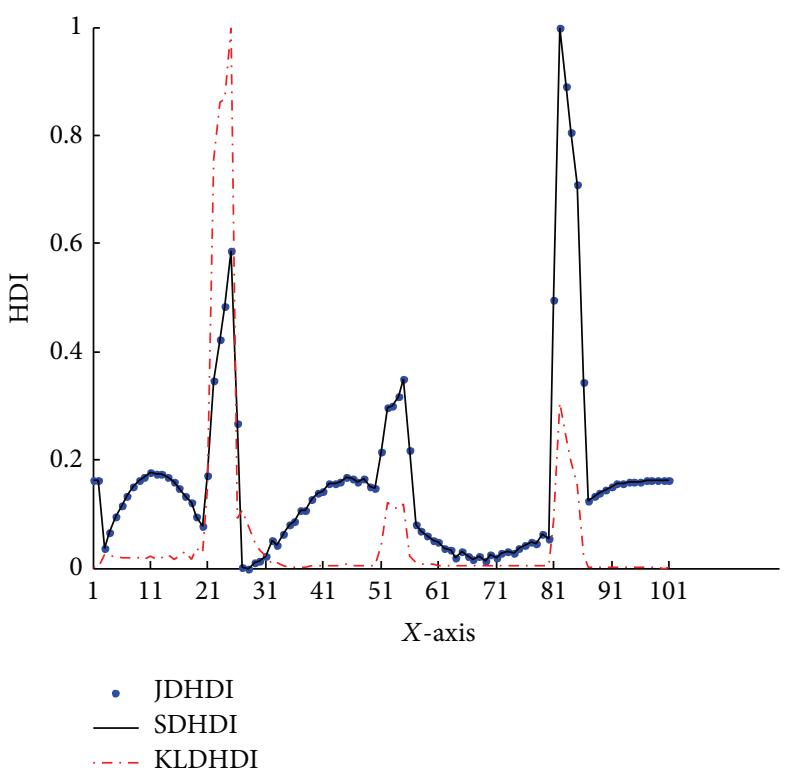

(c) HDI of three kinds of damage

FIGURE 4: Results of three kinds of damage in D1.

the tendency of the HDI is adverse in comparison with the KLD and its approximations; hence the HDI can improve the performance of the KLD in the damage detection.

The damage extent of the first damage scenario is deeper than the second damage scenario; meanwhile, the peak in the damage location in the first damage scenario is larger than the corresponding peak in the second damage scenario, which affects the error of the damage detection.

3.2. Case Study II: Laminated Composite. In this section, an orthotropic laminated composite is employed to demonstrate the effectiveness and robustness of the proposed method.
3.2.1. Material Properties. In this study, a 16-layer square laminated composite with a side length of $240 \mathrm{~mm}$ and a total thickness of $3.2 \mathrm{~mm}$ is considered. The ply orientation distribution along the plate thickness is $\left[0^{\circ} / 0^{\circ} / / 90^{\circ} / 90^{\circ} / 0^{0} / 0^{\circ} / 90^{\circ} / 90^{\circ}\right]_{\mathrm{s}}$, where the "//" denotes the location of the only kind of delamination which lies in the gap between the second layer and the third layer. The material constants of the laminate are $E_{1}=125 \mathrm{GPa}$, $E_{2}=E_{3}=8.5 \mathrm{GPa}, G_{12}=G_{13}=4.5 \mathrm{GPa}, G_{23}=3.27 \mathrm{GPa}$, $v_{12}=v_{13}=v_{23}=0.3$, and $\rho=1550 \mathrm{~kg} \mathrm{~m}^{-3}$.

3.2.2. The Identification of the Delamination. After the delamination occurs in the laminated composite, the delamination 


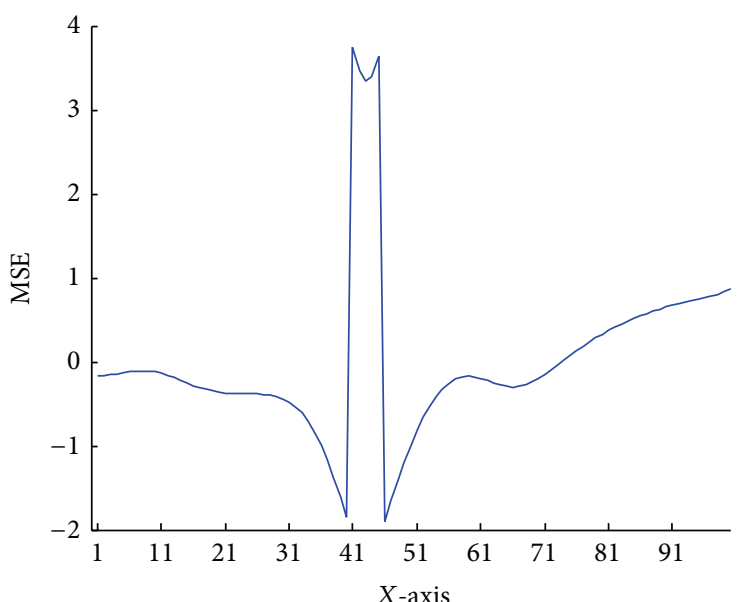

(a) MSE of the single beam

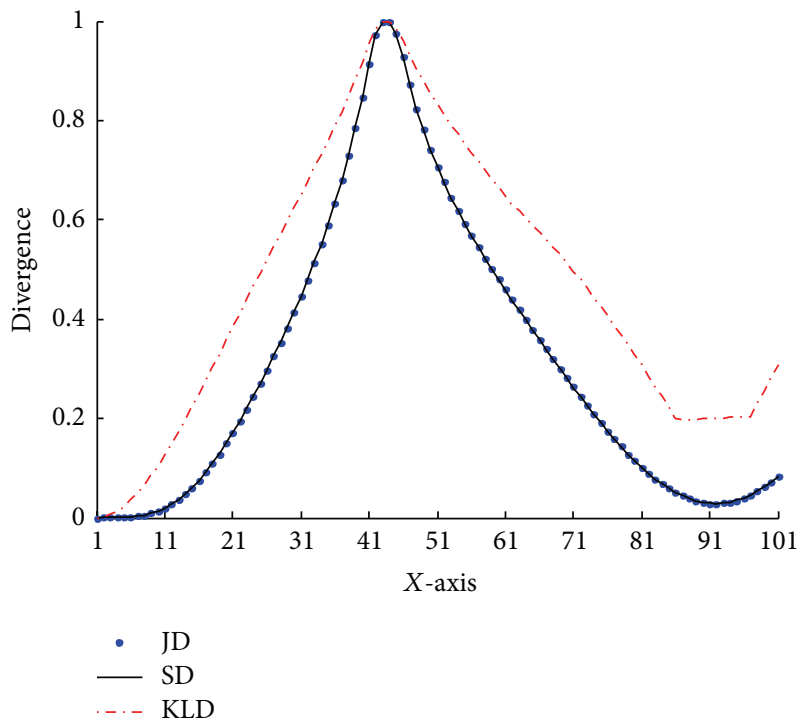

(b) Divergence of the single kind of damage

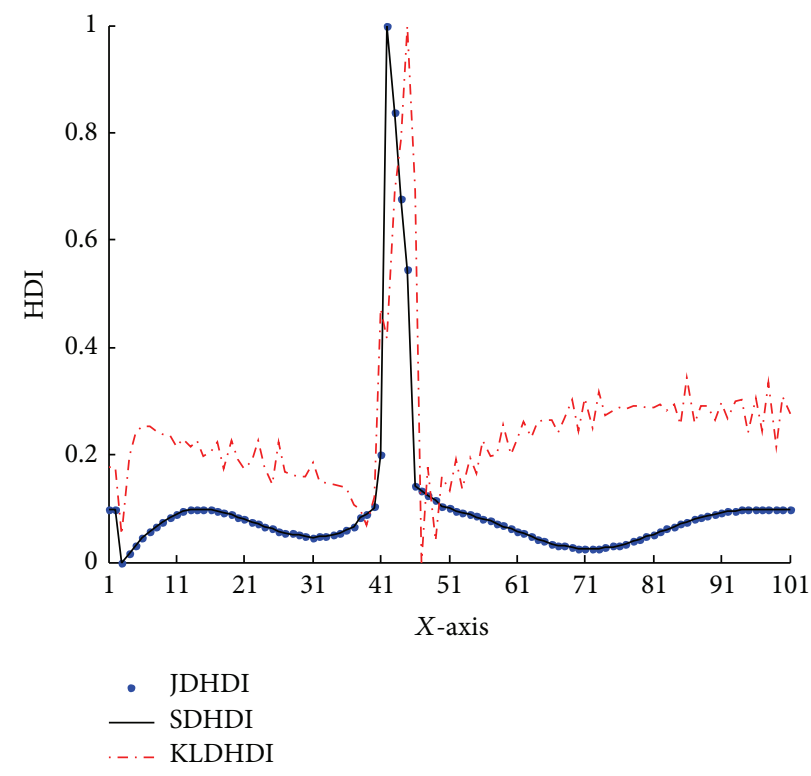

(c) HDI of the single damage

Figure 5: Results of the single kind of damage in D2.

region is between two separate sublaminates, which are called the upper and lower sublaminates, respectively. In this section, the delamination is numerically simulated by the method of merging nodes within the delamination region, which has the same coordinate but belongs to the upper and lower sublaminates.

When the laminated composite is in motion, elements of the upper and lower sublaminates may overlap or even penetrate into each other within the delamination region, whereas it is physically impossible. In order to avoid this phenomenon, the virtual spring element is added between penetrated nodes in the upper and lower sublaminates within the delamination region. And the stiffness coefficient of the virtual spring element is set to 0.1 as same as in [41].
In the delamination scenario 3 , the area of the single delamination is a square with a side length of $100 \mathrm{~mm}$. In addition, the area of two kinds of delamination is both a square and with a side length of $60 \mathrm{~mm}$, and the configuration of three kinds of delamination is all a square with a side length of $40 \mathrm{~mm}$. Furthermore, in the delamination scenario 4, the area of the single delamination is $140 \times 90 \mathrm{~mm}^{2}$, and the areas of two and three kinds of delamination are $60 \times 80 \mathrm{~mm}^{2}$ and $40 \times 70 \mathrm{~mm}^{2}$, respectively, which is shown in Figure 8 .

On the basis of the technique of the modal analysis, the first three mode shapes of the laminated composite before and after the presence of the delamination in the damage scenarios 3 and 4 are obtained, and then the KLD and its 

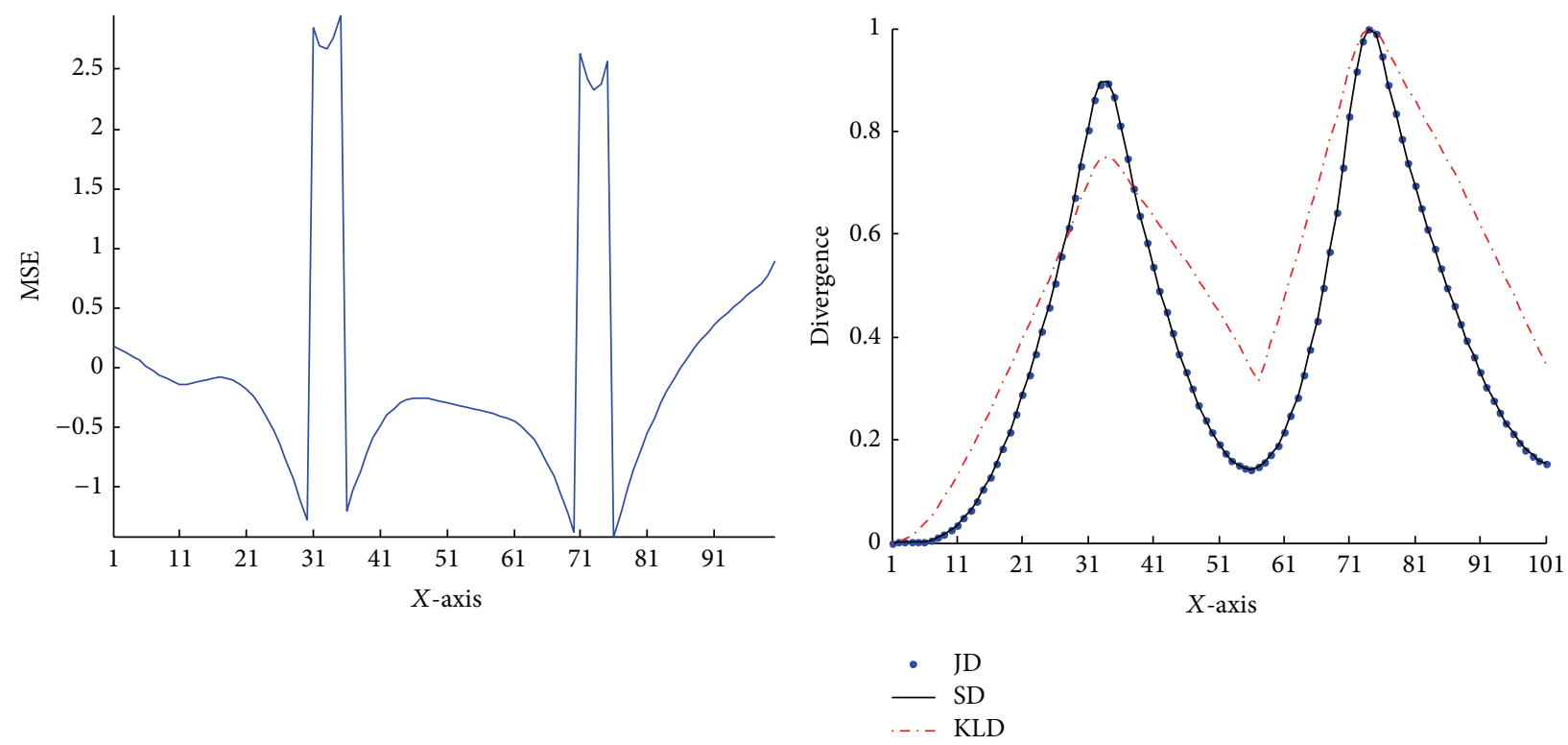

(a) MSE of the single kind of damage

(b) Divergence of two kinds of damage

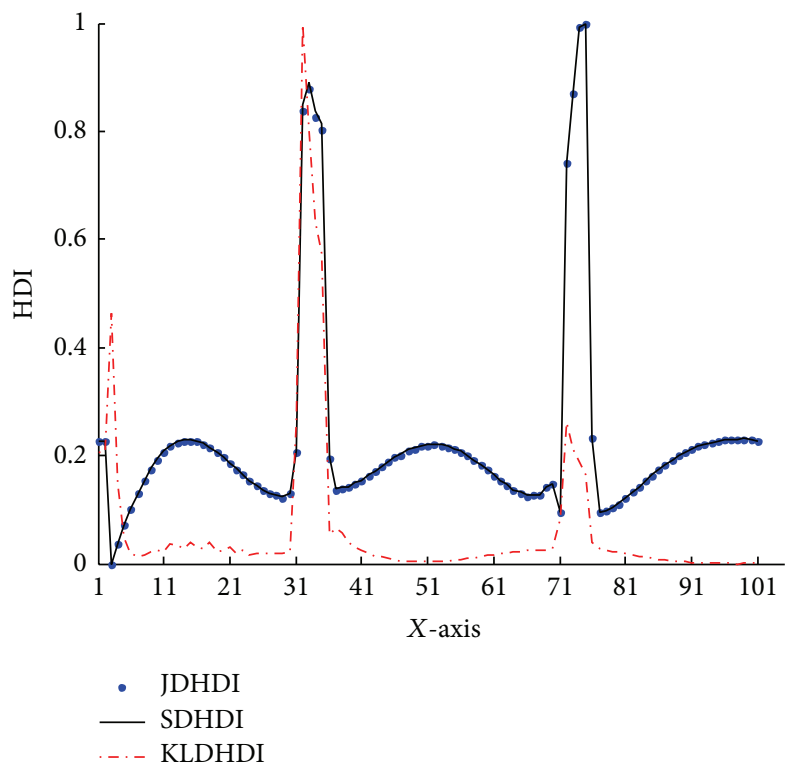

(c) HDI of two kinds of damage

FIgURE 6: Results of two kinds of damage in D2.

approximation are calculated. In order to reduce and remove the error, the hybrid damage index based on the KLD and its approximation is applied with the intension of identifying the delamination in laminated composite more accurately, and the method of modal strain energy is employed so as to verify the efficiency of the proposed method; finally, all results of the delamination identification are shown in Figures 9, 10, 11, 12, 13 , and 14.

3.2.3. Discussions. The presence and location of the delamination can be obtained through the sudden change of the corresponding curve; thus it can come to the conclusion that the KLD and its approximations are able to identify all the delamination in the delamination scenarios 3 and 4. The MSE is able to identify the single delamination and two kinds of delamination, whereas it is not able to identify three kinds of delamination in the delamination scenarios 3 and 4. Furthermore, the HDI based on the KLD and its approximation is able to identify all the delamination in the delamination scenarios 3 and 4 . As a consequence, the HDI based on the KLD and its approximations is more sensitive to the delamination than the MSE.

It is worth noting out that the error of the delamination identification by the KLD and its approximation is relatively 


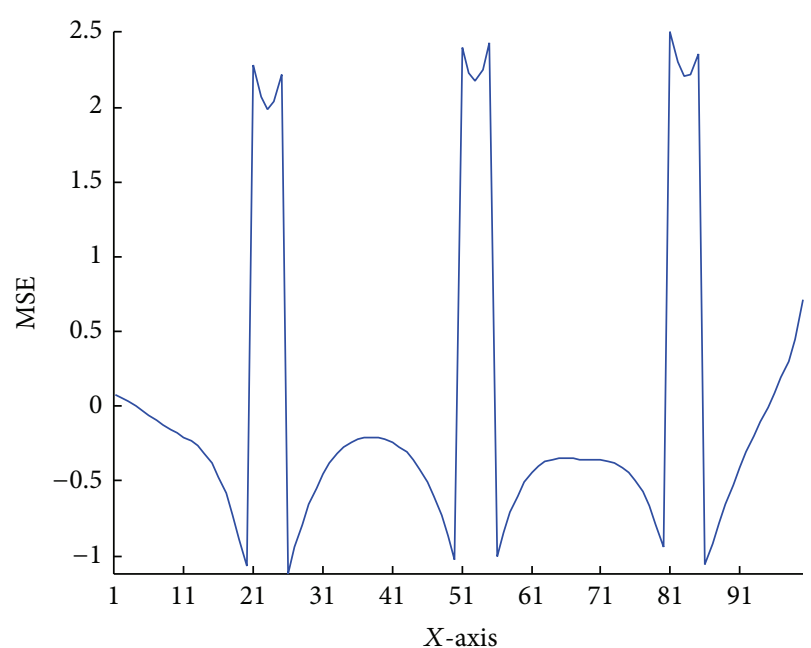

(a) MSE of the single kind of damage

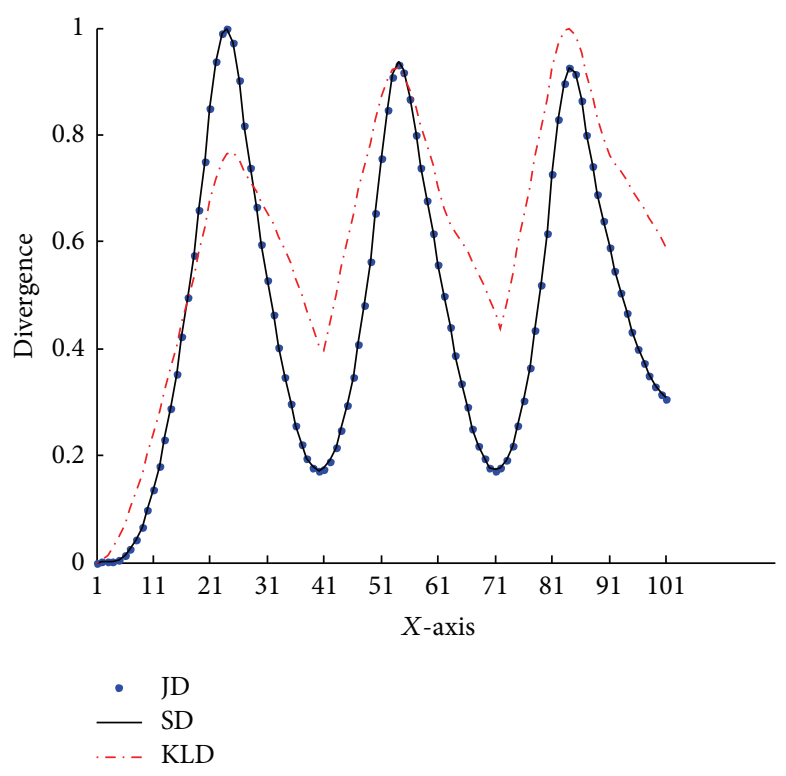

(b) Divergence of three kinds of damage

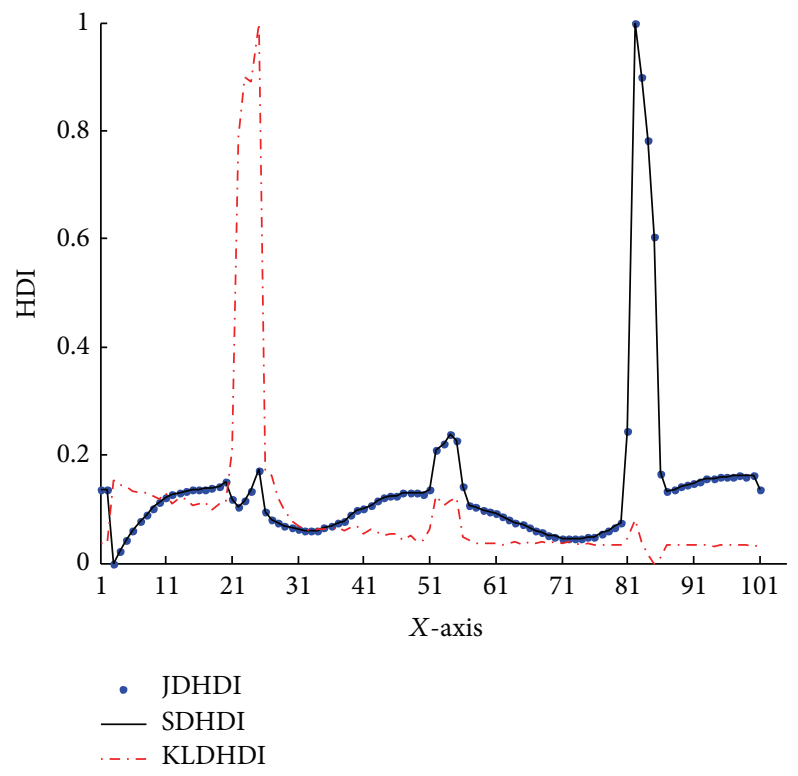

(c) HDI of three kinds of damage

FIGURE 7: Results of three kinds of damage in D2.

large. The JD and $\chi^{2}$ distribution have a much larger error in identifying the single and two kinds of delamination, and values in the boundary of the laminated composite are relatively large in comparison with peaks in the location of the delamination. In addition, values in the boundary are even larger than the value in the delamination location when the JD and $\chi^{2}$ distribution are employed to identify the single delamination. Generally speaking, this will reduce the ability of the KLD and its approximations to identify the delamination.

The MSE and HDI based on the KLD and its approximations are both able to identify the single delamination in the delamination scenario 3, whereas the HDI based on the KLD (KLDHDI) has a relative large error. The reason for this is that the values at $x=200 \mathrm{~mm}$ are especially large, and this can reduce the sensitivity of the KLDHDI to the delamination.

The MSE have a much larger error of the delamination identification in comparison with the HDI when they are employed to identify two kinds of delamination in the delamination scenario 4. From Figure 13(g) we can know that the value in the same boundary is much larger than the peak in the delamination location. As a consequence, the MSE have a weaker ability of identifying delamination than the HDI based on the KLD and its approximation. 


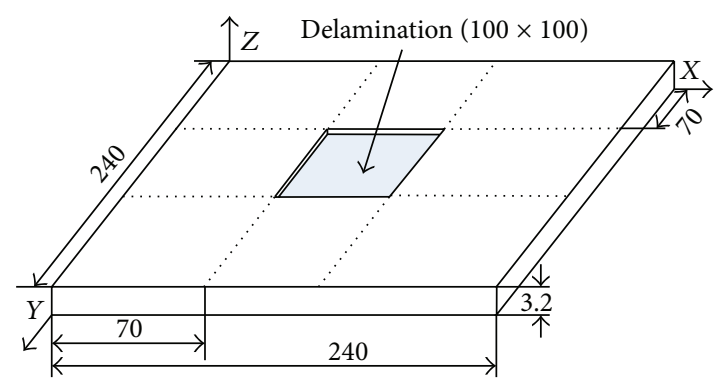

(a) Delamination scenario of the single delamination

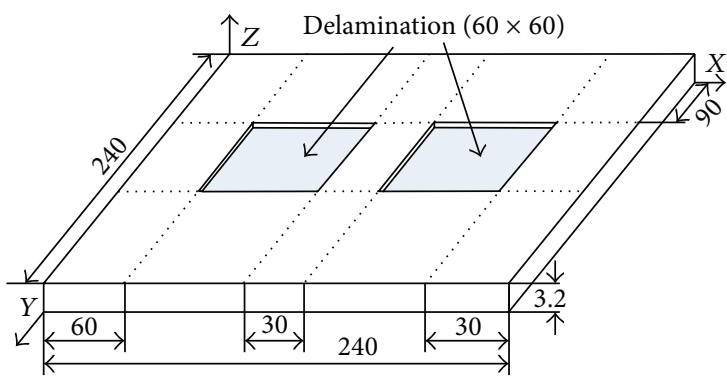

(b) Delamination scenario of two kinds of delamination

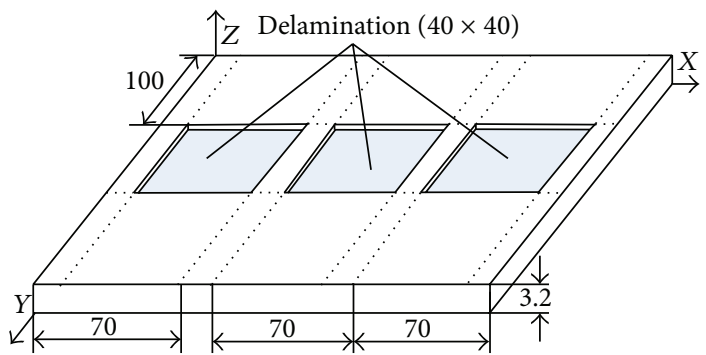

(c) Delamination scenario of three kinds of delamination

Figure 8: Delamination scenarios of the laminated composite.

The value of the HDI based on the KLD and its approximations in the region between two kinds of delamination is nearly zero when it is employed to identify two kinds of delamination, whereas the corresponding value of the MSE is much larger. It is found that the HDI have a better ability than the MSE in identifying two kinds of delamination.

However, the value of the HDI changes much in different delamination locations, when it is employed to identify multiple kinds of delamination, and it is a direction that needs improvement of the HDI based on the KLD and its approximations.

Generally speaking, it is worth pointing out that the HDI have a better ability to identify delamination than the MSE.

3.2.4. Addition of the Measurement Noise. For the real modal testing, it is expected that there would be some deviations due to measurement noise. In order to study the efficiency of the proposed method in the noisy environment, the noise is added with the method in [42]. For simplicity, the identification of three kinds of delamination by HDI based on the JD is employed to study the effect of the noise, and four levels $(5 \%, 10 \%, 15 \%$ and $20 \%)$ of noise are added to the raw of the first three mode shapes. Finally, the result of the delamination identification is shown in Figure 15.

From the result it is found that the noise mainly affects the value of JDHDI in the intact region of the laminated composite. When the amount of the noise is 5 percent, JDHDI varies a little greater than the one with noise absent in the intact region, whereas the JDHDI is almost the same in the delamination region; the analogous conclusion also can be obtained with another level of noise. Furthermore, the change of the JDHDI becomes greater in the undelaminationed region, as the amount of noise increases; meanwhile the relative large percent (20) of noise is added, three kinds of delamination can still be identified in high accuracy, and it demonstrates that the proposed method is less sensitive to the noise.

It indicates that the noise mainly affects the error of the delamination in a permissible tolerance, and the results of the delamination identification give a reasonable agreement between the damage identified and the damage assumed. Finally, it is found that the proposed method is an effective and robust method for the delamination identification.

\section{Conclusions}

In this paper the hybrid damage index for structural damage detection based on the KLD and its approximations has been proposed, and the following conclusions can be drawn from the above study.

(1) The KLD and its approximations are able to detect the damage in all damage scenarios in this paper, whereas the error of the damage identification is relatively large. As a consequence, the KLD and its approximations are suitable for the beam and the laminated composite.

(2) The localized ability on the damage location of the hybrid damage index is stronger than the KLD and its approximations, and the corresponding error of the damage detection by the hybrid damage index is still less than the KLD and its approximations. Hence the hybrid damage index outperforms the corresponding KLD and its approximation. 


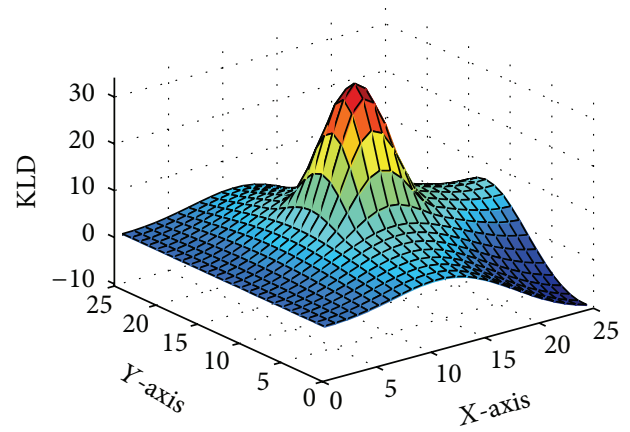

(a) KLD of the single kind of delamination

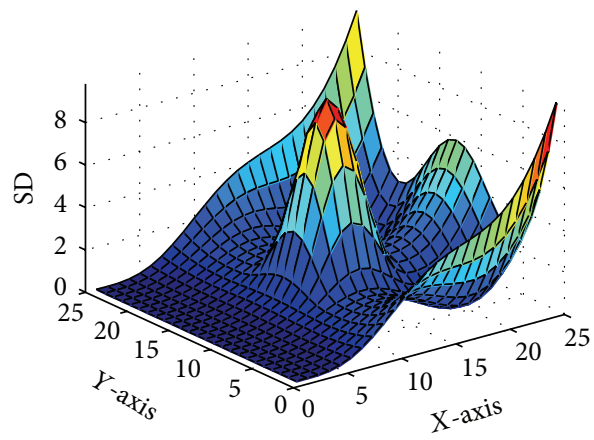

(c) SD of the single kind of delamination

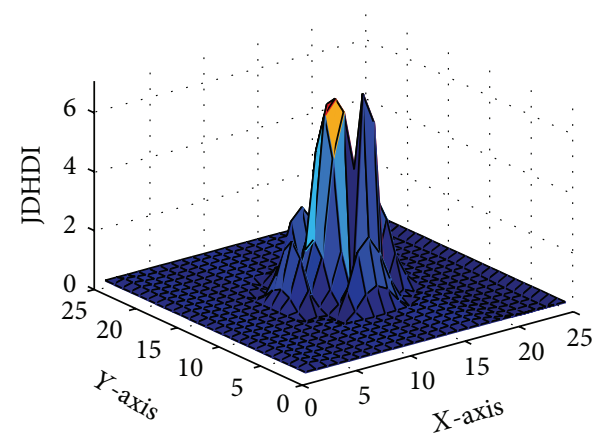

(e) JDHDI of the single kind of delamination

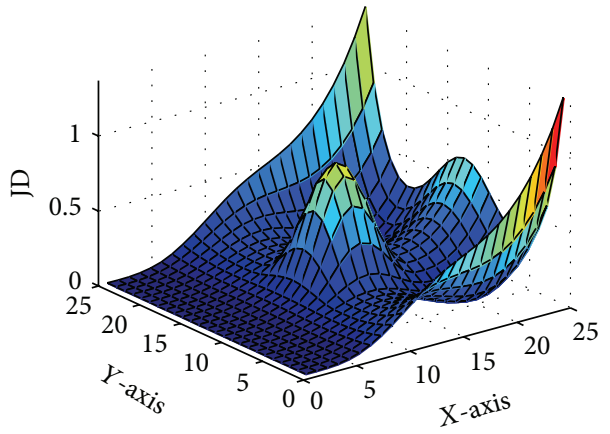

(b) JD of the single kind of delamination

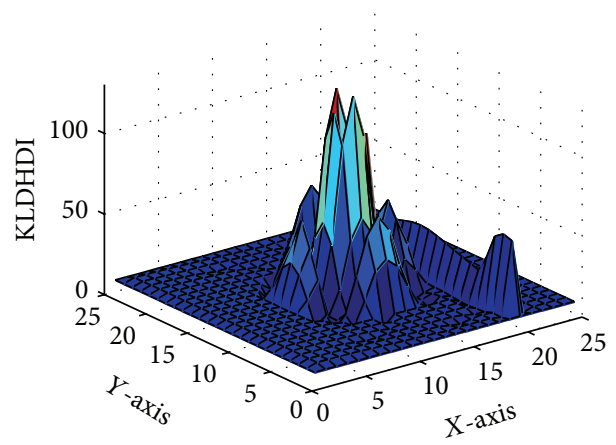

(d) KLDHDI of the single kind of delamination

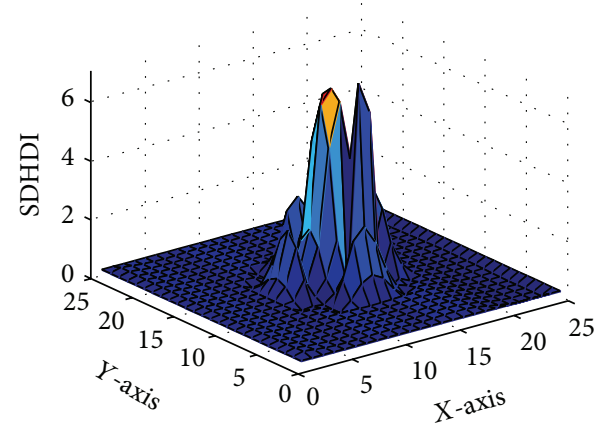

(f) SDHDI of the single kind of delamination

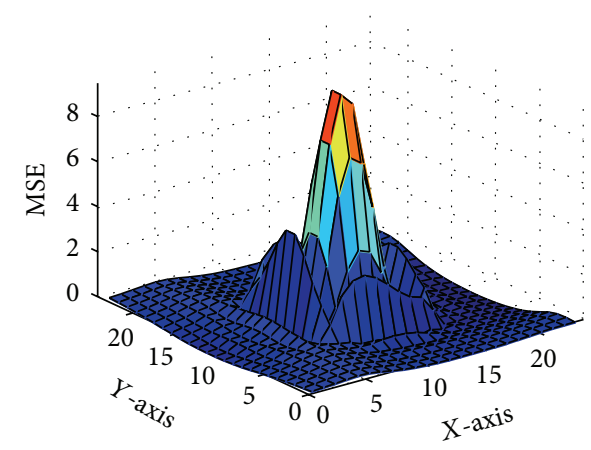

(g) MSE of the single kind of delamination

FIGURE 9: Results of the single kind of delamination identification in D3. 


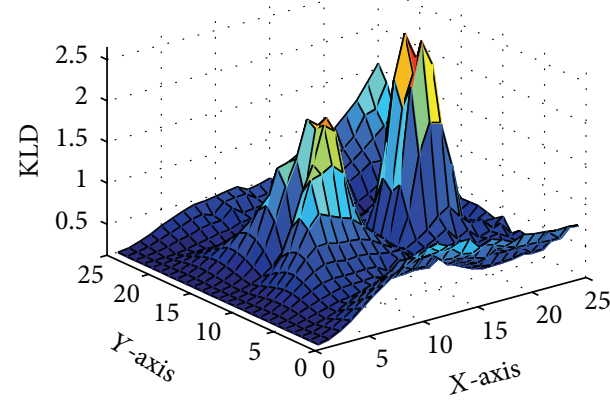

(a) KLD of two kinds of delamination

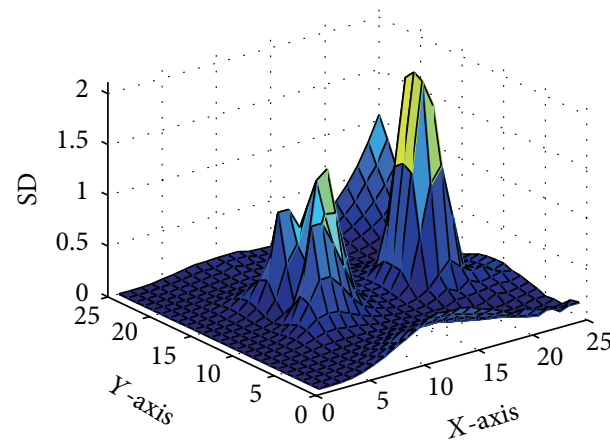

(c) SD of two kinds of delamination

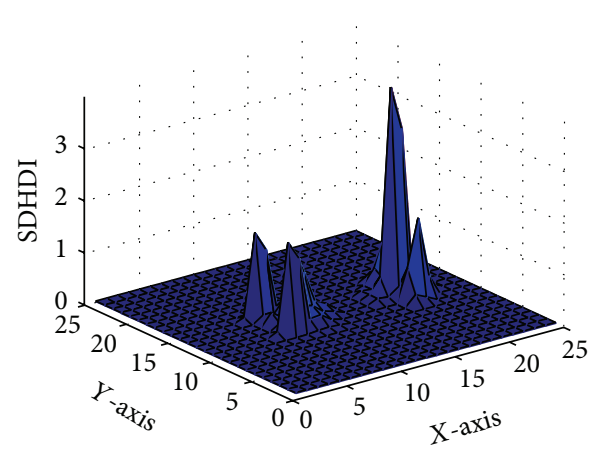

(e) JDHDI of two kinds of delamination

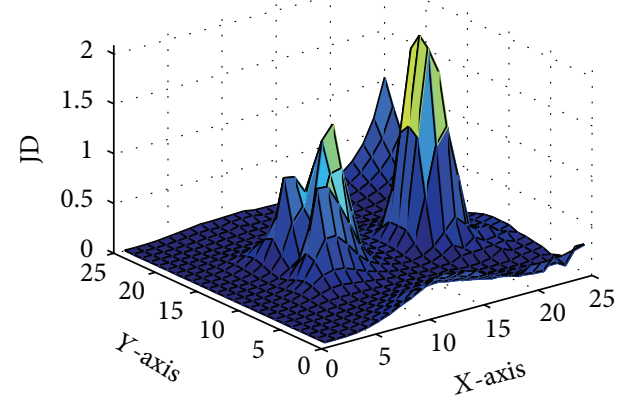

(b) JD of two kinds of delamination

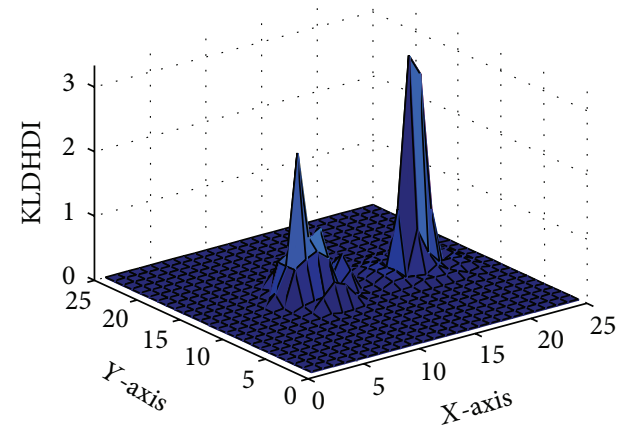

(d) KLDHDI of two kinds of delamination

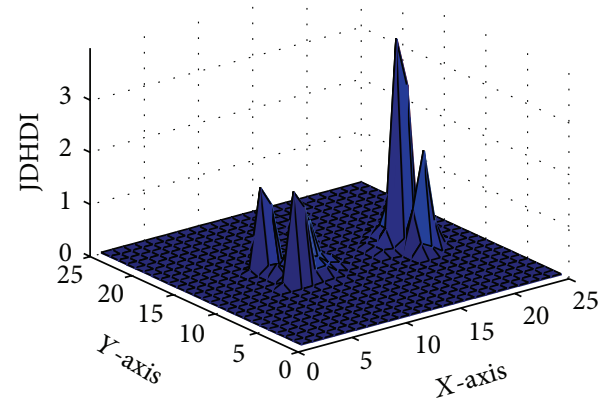

(f) SDHDI of two kinds of delamination

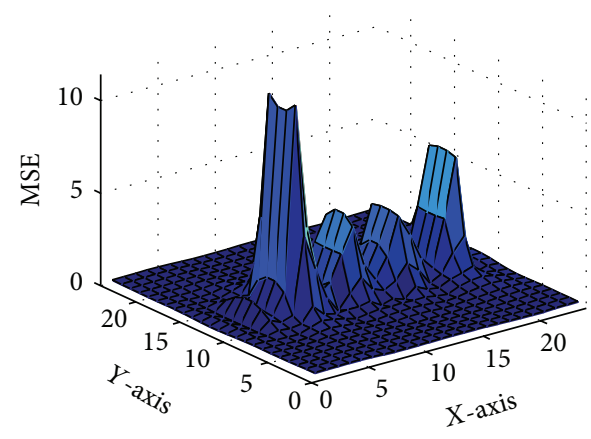

(g) MSE of two kinds of delamination

Figure 10: Results of the single kind of delamination identification in D3. 


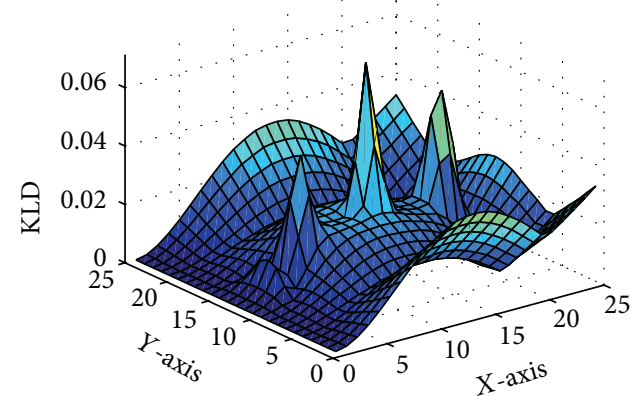

(a) KLD of three kinds of delamination

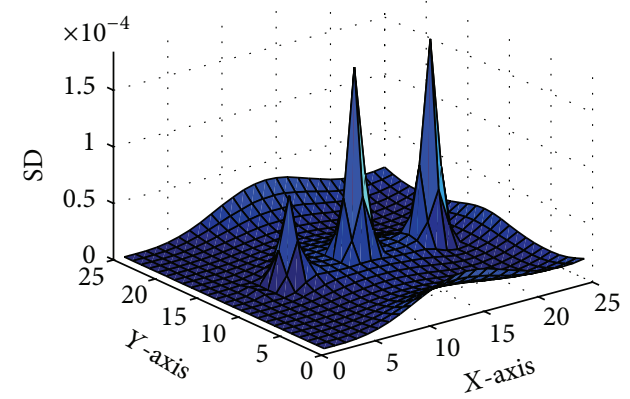

(c) SD of the single kind of delamination

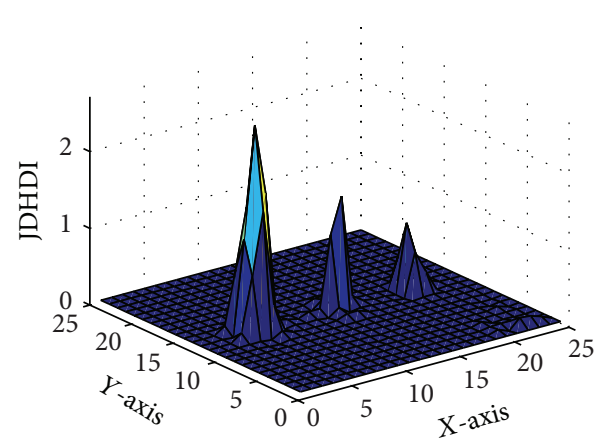

(e) JDHDI of the single kind of delamination

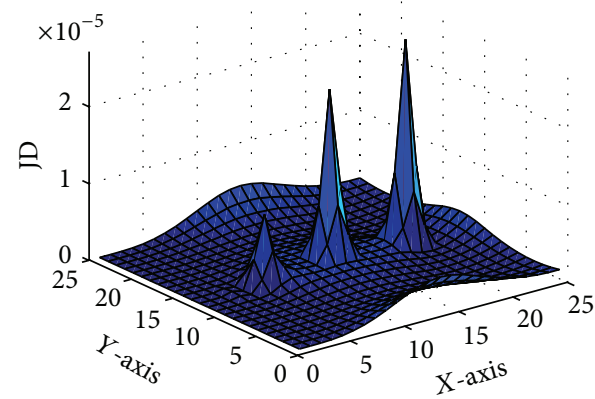

(b) JD of three kinds of delamination

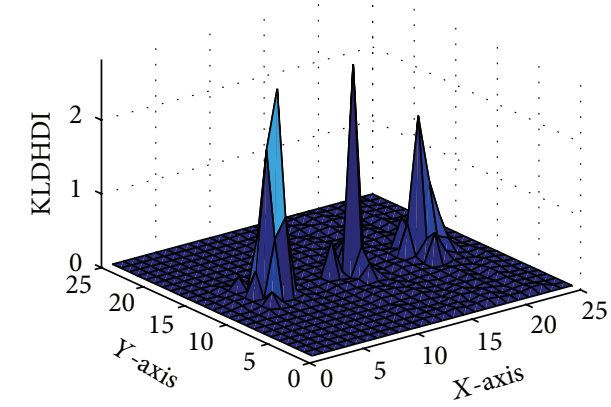

(d) KLDHDI of the single kind of delamination

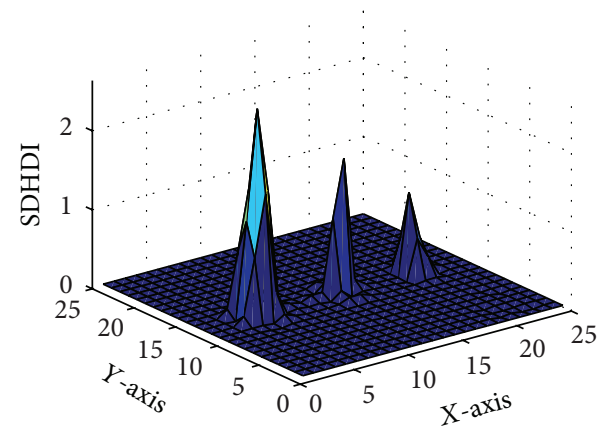

(f) SDHDI of the single kind of delamination

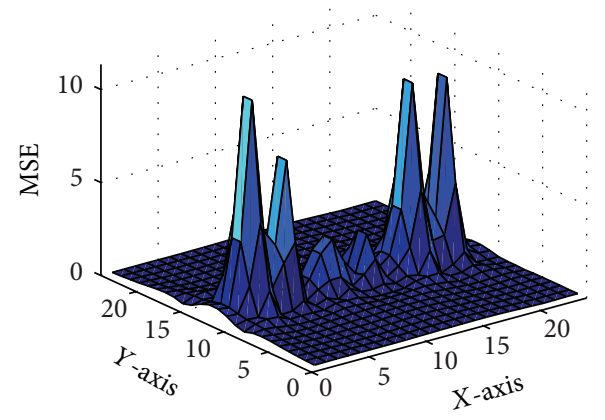

(g) MSE of the single kind of delamination

Figure 11: Results of the delamination identification in D3. 


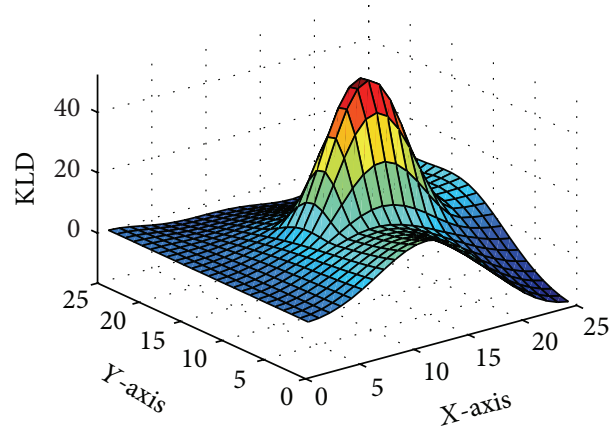

(a) KLD of the single kind of delamination

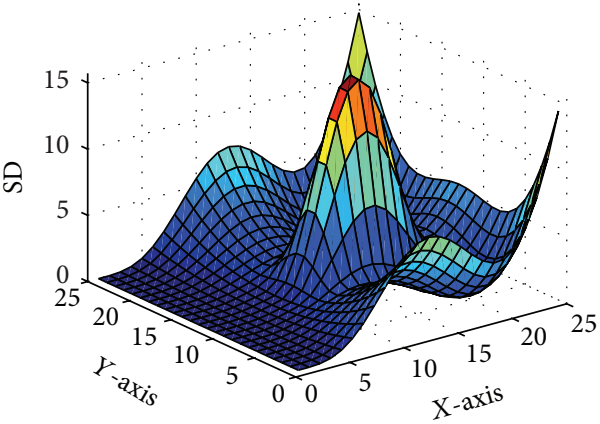

(c) SD of the single kind of delamination

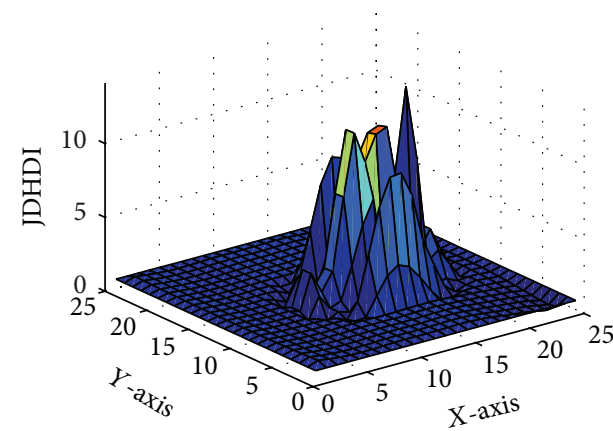

(e) JDHDI of the single kind of delamination

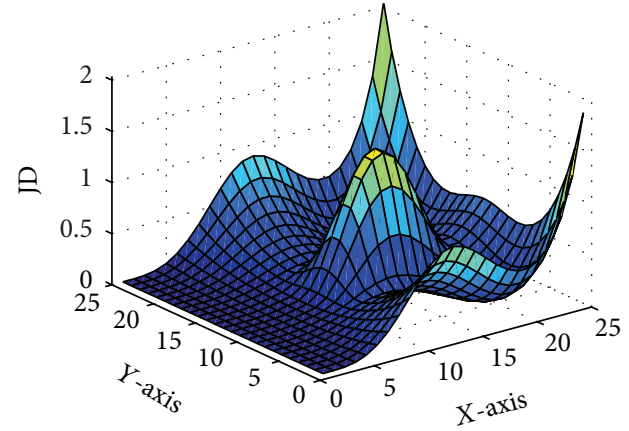

(b) JD of the single kind of delamination

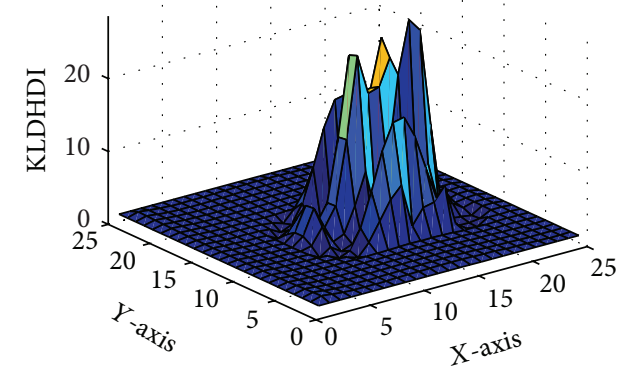

(d) KLDHDI of the single kind of delamination

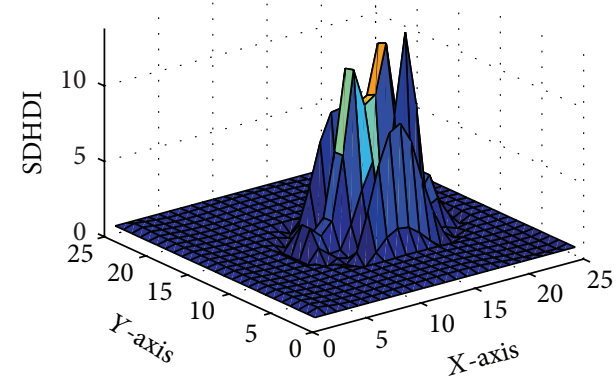

(f) SDHDI of the single kind of delamination

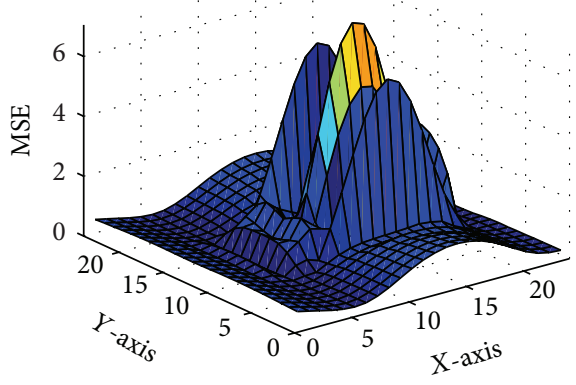

(g) MSE of the single kind of delamination

FIGURE 12: Results of the single kind of delamination identification in D4. 


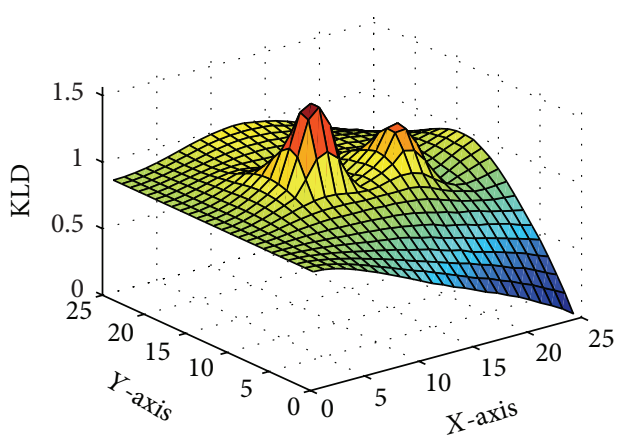

(a) KLD of two kinds of delamination

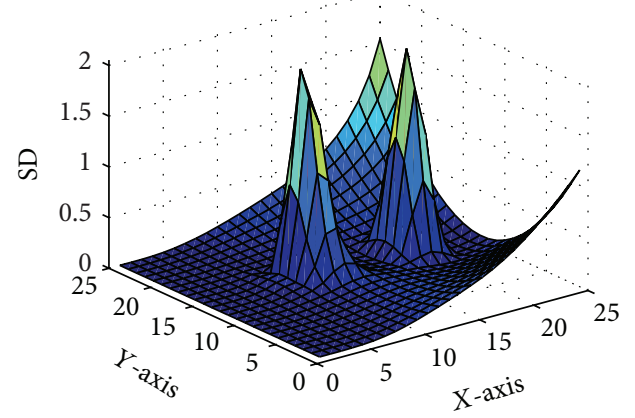

(c) SD of two kinds of delamination

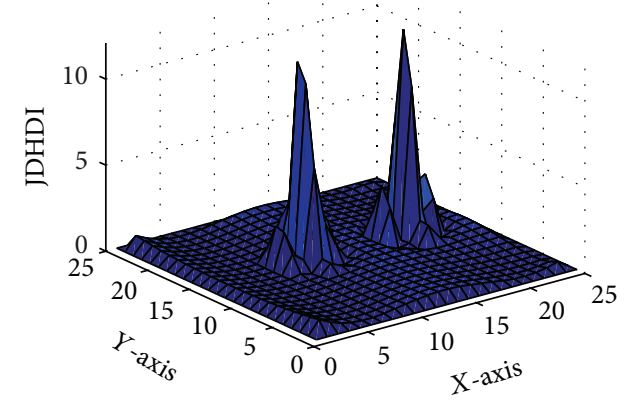

(e) JDHDI of two kinds of delamination

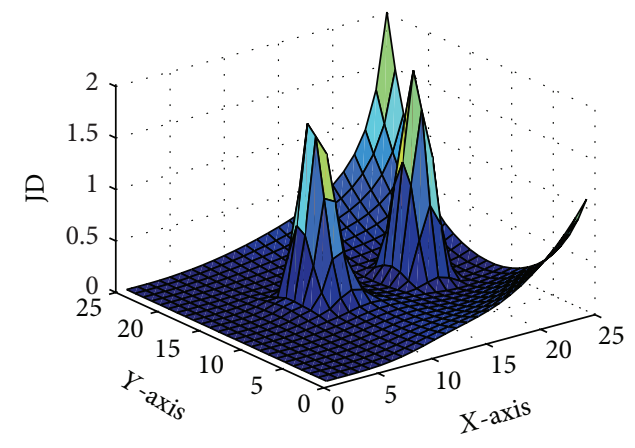

(b) JD of two kinds of delamination

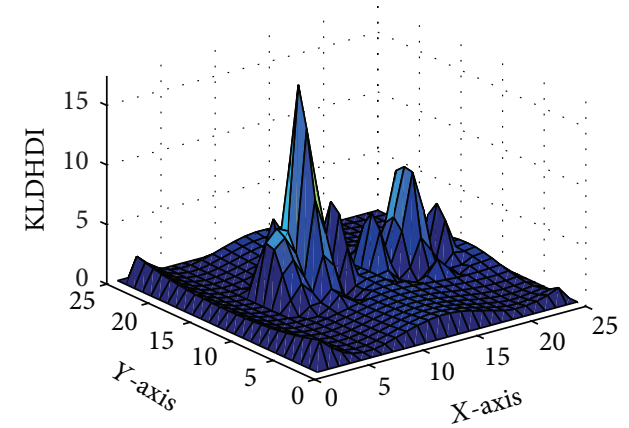

(d) KLDHDI of two kinds of delamination

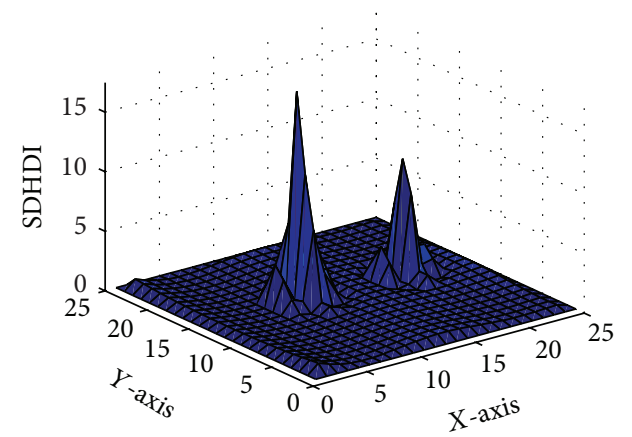

(f) SDHDI of two kinds of delamination

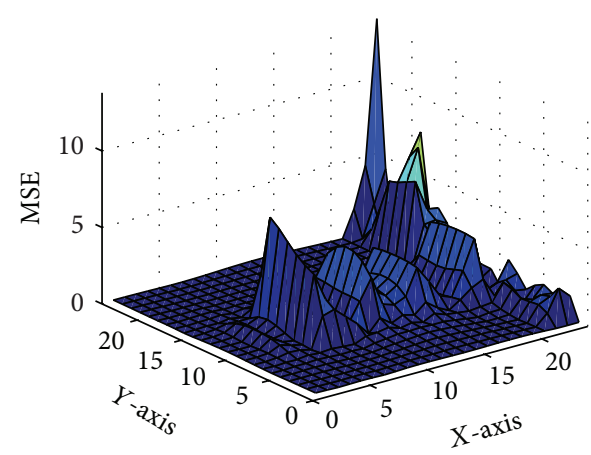

(g) MSE of two kinds of delamination

FIGURE 13: Results of two kinds of delamination identification in D4. 


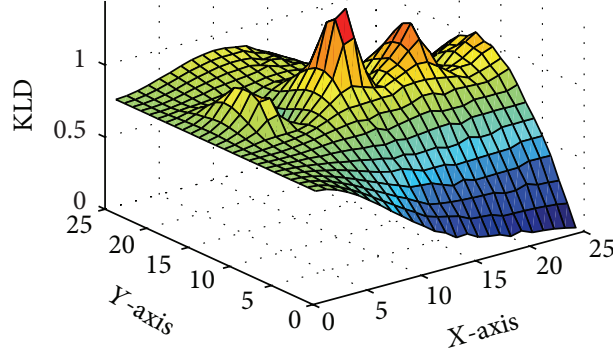

(a) KLD of three kinds of delamination

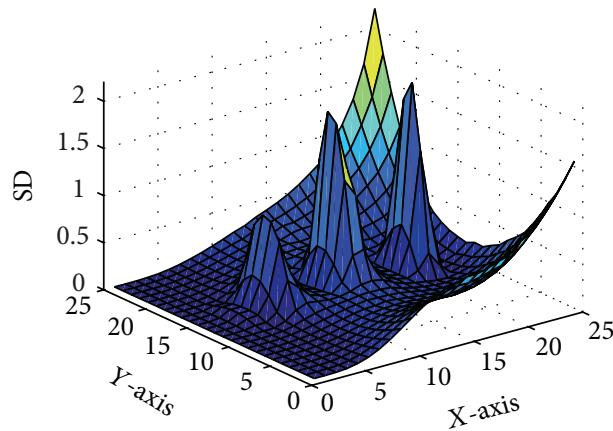

(c) SD of three kinds of delamination

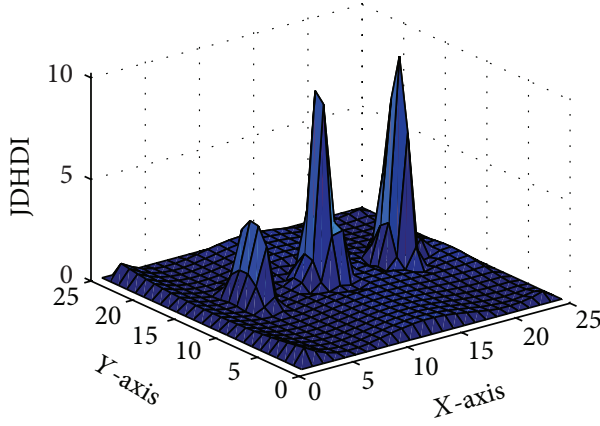

(e) JDHDI of two kinds of delamination

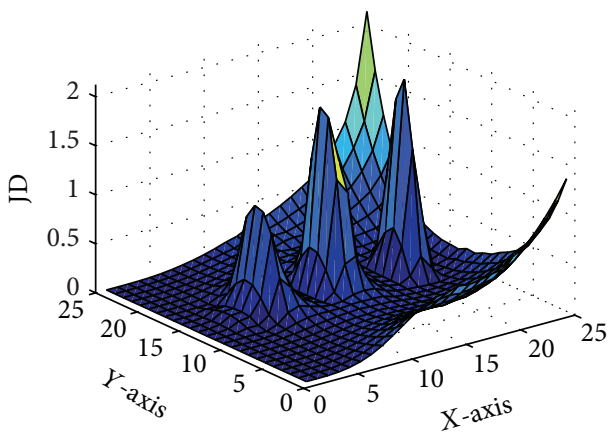

(b) JD of three kinds of delamination

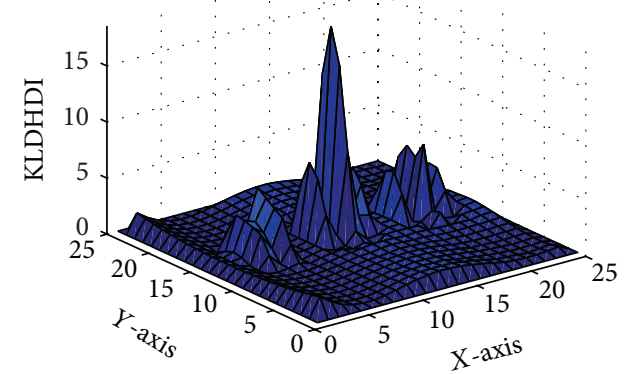

(d) KLDHDI of three kinds of delamination

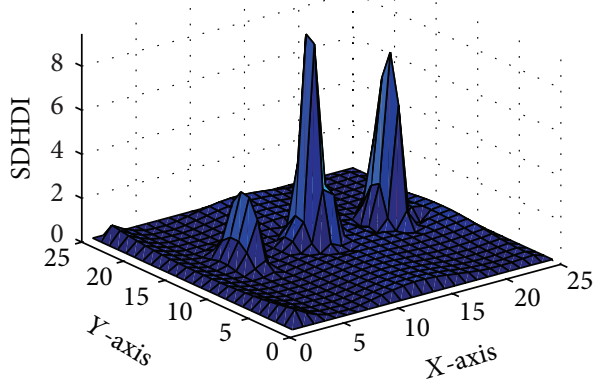

(f) SDHDI of two kinds of delamination

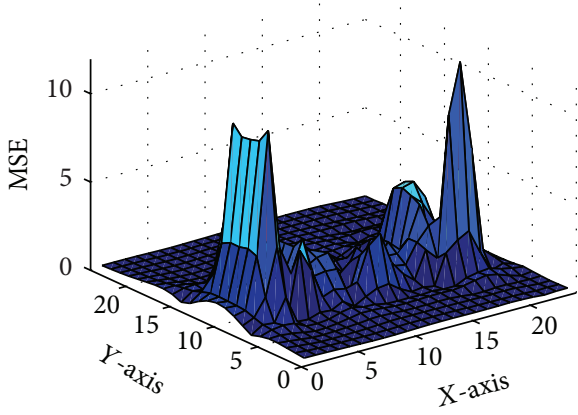

(g) MSE of three kinds of delamination

FIGURE 14: Results of two kinds of delamination identification in D4. 


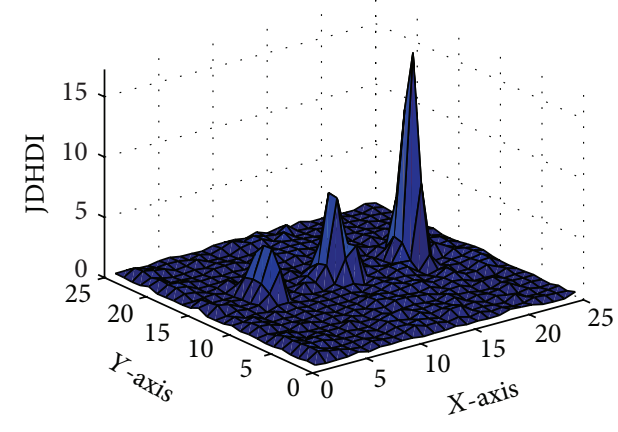

(a) JDHDI of three kinds of delamination with $5 \%$ noise

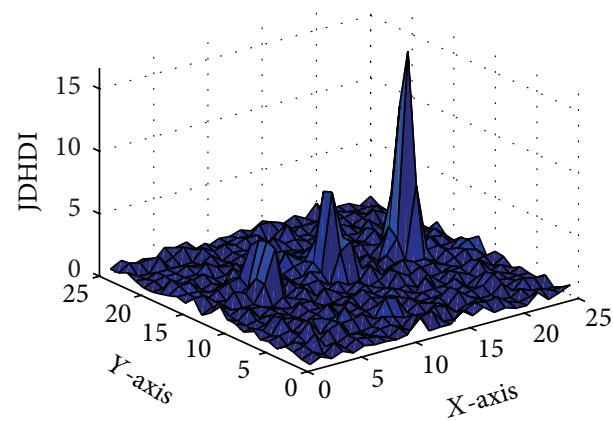

(c) JDHDI of three kinds of delamination with 15\% noise

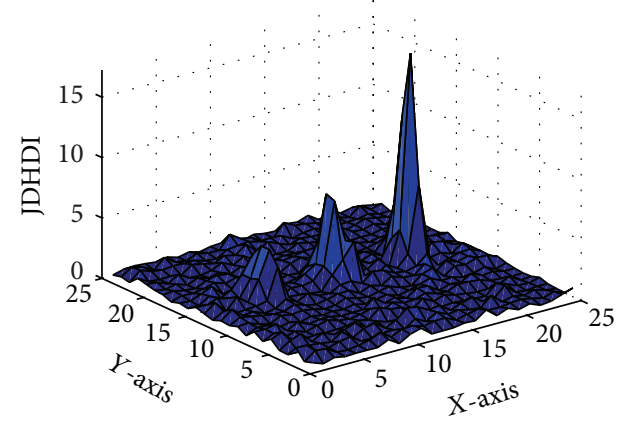

(b) JDHDI of three kinds of delamination with 10\% noise

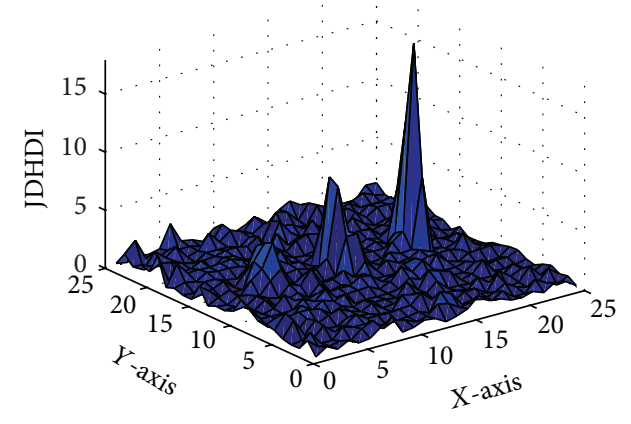

(d) JDHDI of three kinds of delamination with $20 \%$ noise

FIGURE 15: JDHDI with noise.

(3) The MSE method is not able to detect three kinds of delamination for the laminated composite; meanwhile the corresponding error of the damage detection is relatively large, whereas the hybrid damage index based on the KLD and its approximations not only is able to detect the damage in all damage scenarios, but also induces a small error of the damage identification. Thus the hybrid damage index based on the KLD and its approximations is a better damage index in comparison with the modal strain energy method.

(4) The applicability of the KLD and hybrid damage index to different types of structures and different sizes of damage is under experimental validation and it will be illustrated in the future work.

(5) Results of the present work refer to the beam and laminated composite; moreover they can be easily extended to more complex structures and more complicated boundary conditions.

In conclusion, the present consequences provide a foundation of using the hybrid damage index based on the KLD and its approximations as an efficient tool in the damage and delamination identification in the beam and laminated composite, respectively. Moreover, the enhancement for reliability and precision of the proposed method is undergoing in our next research.

\section{Conflict of Interests}

The authors declare that there is no conflict of interests regarding the publication of this paper.

\section{Acknowledgments}

The research described in this paper has been supported by the National Natural Science Foundation of China (Grant nos. 51035007 and 51175401) and the Research Fund for the Doctoral Program of Higher Education of China (no. 20110201130001).

\section{References}

[1] G. Park, H. Sohn, C. R. Farrar, and D. J. Inman, "Overview of piezoelectric impedance-based health monitoring and path forward," Shock and Vibration Digest, vol. 35, no. 6, pp. 451-463, 2003.

[2] G. Park, H. H. Cudney, and D. J. Inman, "Impedance-based health monitoring of civil structural components," Journal of Infrastructure Systems, vol. 6, no. 4, pp. 153-160, 2000.

[3] A. S. Sekhar, "Multiple cracks effects and identification," Mechanical Systems and Signal Processing, vol. 22, no. 4, pp. 845878, 2008.

[4] W. Fan and P. Qiao, "Vibration-based damage identification methods: a review and comparative study," Structural Health Monitoring, vol. 10, no. 1, pp. 83-111, 2011. 
[5] Y. Y. Li, "Hypersensitivity of strain-based indicators for structural damage identification: a review," Mechanical Systems and Signal Processing, vol. 24, no. 3, pp. 653-664, 2010.

[6] J. M. Nichols, S. T. Trickey, and M. Seaver, "Damage detection using multivariate recurrence quantification analysis," Mechanical Systems and Signal Processing, vol. 20, no. 2, pp. 421-437, 2006.

[7] C. Sun, Z. Zhang, W. Cheng et al., "Manifold subspace distance derived from kernel principal angles and its application to machinery structural damage assessment," Smart Materials and Structures, vol. 22, no. 8, Article ID 085012, 2013.

[8] A.-M. Yan and J.-C. Golinval, "Null subspace-based damage detection of structures using vibration measurements," Mechanical Systems and Signal Processing, vol. 20, no. 3, pp. 611-626, 2006.

[9] D. Bueno and J.J. Sinou, "Structural damage identification and location using grammian matrices," Shock and Vibration, vol. 19, no. 3, pp. 287-299, 2012.

[10] J. Xiang, T. Matsumoto, Y. Wang et al., "Detect damages in conical shells using curvature mode shape and wavelet finite element method," International Journal of Mechanical Sciences, vol. 66, pp. 83-93, 2013.

[11] S. Wang, W. Huang, and Z. K. Zhu, “Transient modeling and parameter identification based on wavelet and correlation filtering for rotating machine fault diagnosis," Mechanical Systems and Signal Processing, vol. 25, no. 4, pp. 1299-1320, 2011.

[12] Z. Yang, X. Chen, J. Yu et al., "A damage identification approach for plate structures based on frequency measurements," Nondestructive Testing and Evaluation, vol. 28, no. 4, pp. 1-21, 2013.

[13] X. Liu, Z. Jiang, and Z. Yan, "Improvement of accuracy in damage localization using frequency slice wavelet transform," Shock and Vibration, vol. 19, no. 4, pp. 585-596, 2012.

[14] S. M. Nalawade, N. Mahra, K. T. V. Grattan, and H. V. Thakur, "Delamination detection in glass composites using embedded Hi-Bi photonic crystal fiber," Smart Materials and Structures, vol. 20, no. 5, Article ID 055023, 2011.

[15] D. Wang, L. Ye, Z. Su, and Y. Lu, "Quantitative identification of multiple damage in laminated composite beams using A0 Lamb mode," Journal of Composite Materials, vol. 45, no. 20, pp. 20612069, 2011.

[16] D. Wang, L. Ye, Y. Lu, and F. Li, "A damage diagnostic imaging algorithm based on the quantitative comparison of Lamb wave signals," Smart Materials and Structures, vol. 19, no. 6, Article ID 065008, 2010.

[17] Z. Yang, L. Wang, H. Wang, Y. Ding, and X. Dang, "Damage detection in composite structures using vibration response under stochastic excitation," Journal of Sound and Vibration, vol. 325, no. 4-5, pp. 755-768, 2009.

[18] S. Shang, G. J. Yun, and P. Qiao, "Delamination identification of laminated composite plates using a continuum damage mechanics model and subset selection technique," Smart Materials and Structures, vol. 19, no. 5, Article ID 055024, 2010.

[19] H. S. Kim, J. Kim, S.-B. Choi, A. Ghoshal, and A. Chattopadhyay, "Modal-strain-based damage index of laminated composite structures using smooth transition of displacements," AIAA Journal, vol. 45, no. 12, pp. 2972-2978, 2007.

[20] Y. Liu, S. Mohanty, and A. Chattopadhyay, "Condition based structural health monitoring and prognosis of composite structures under uniaxial and biaxial loading," Journal of Nondestructive Evaluation, vol. 29, no. 3, pp. 181-188, 2010.
[21] Y. Liu, M. Y. Fard, A. Chattopadhyay, and D. Doyle, "Damage assessment of CFRP composites using a time-frequency approach," Journal of Intelligent Material Systems and Structures, vol. 23, no. 4, pp. 397-413, 2012.

[22] K. Worden and A. P. Burrows, "Optimal sensor placement for fault detection," Engineering Structures, vol. 23, no. 8, pp. 885901, 2001.

[23] Y. G. Xu, G. R. Liu, Z. P. Wu, and X. M. Huang, "Adaptive multilayer perceptron networks for detection of cracks in anisotropic laminated plates," International Journal of Solids and Structures, vol. 38, no. 32-33, pp. 5625-5645, 2001.

[24] G. R. Liu, Z. Wang, G. Y. Zhang, Z. Zong, and S. Wang, "An edge-based smoothed point interpolation method for material discontinuity," Mechanics of Advanced Materials and Structures, vol. 19, no. 1-3, pp. 3-17, 2012.

[25] M. T. Valoor and K. Chandrashekhara, "A thick compositebeam model for delamination prediction by the use of neural networks," Composites Science and Technology, vol. 60, no. 9, pp. $1773-1779,2000$.

[26] M. J. Katz, "Fractals and the analysis of waveforms," Computers in Biology and Medicine, vol. 18, no. 3, pp. 145-156, 1988.

[27] Q. Wang and X. Deng, "Damage detection with spatial wavelets," International Journal of Solids and Structures, vol. 36, no. 23, pp. 3443-3468, 1999.

[28] D. Wu and S. S. Law, "Damage localization in plate structures from uniform load surface curvature," Journal of Sound and Vibration, vol. 276, no. 1-2, pp. 227-244, 2004.

[29] D. Wu and S. S. Law, "Crack identification in thin plates with anisotropic damage model and vibration measurements," Journal of Applied Mechanics, Transactions ASME, vol. 72, no. 6, pp. 852-861, 2005.

[30] X. Y. Li and S. S. Law, "Structural damage detection with statistical analysis from support excitation," Mechanical Systems and Signal Processing, vol. 22, no. 8, pp. 1793-1808, 2008.

[31] Y. Lu, L. Ye, Z. Su, L. Zhou, and L. Cheng, "Artificial Neural Network (ANN)-based crack identification in aluminum plates with lamb wave signals," Journal of Intelligent Material Systems and Structures, vol. 20, no. 1, pp. 39-49, 2009.

[32] M. Radzieński, M. Krawczuk, and M. Palacz, "Improvement of damage detection methods based on experimental modal parameters," Mechanical Systems and Signal Processing, vol. 25, no. 6, pp. 2169-2190, 2011.

[33] J. R. Hershey and P. A. Olsen, "Approximating the Kullback Leibler divergence between Gaussian mixture models," in Proceedings of the IEEE International Conference on Acoustics, Speech and Signal Processing (ICASSP '07), pp. IV317-IV320, April 2007.

[34] S. Eguchi and J. Copas, "Interpreting Kullback-Leibler divergence with the Neyman-Pearson lemma," Journal of Multivariate Analysis, vol. 97, no. 9, pp. 2034-2040, 2006.

[35] A. Smith, P. A. Naik, and C.-L. Tsai, "Markov-switching model selection using Kullback-Leibler divergence," Journal of Econometrics, vol. 134, no. 2, pp. 553-577, 2006.

[36] J. Mathiassen, A. Skavhaug, and K. Bø, “Texture similarity measure using Kullback-Leibler divergence between gamma distributions," in Proceedings of the European Conference on Computer Vision (ECCV '02), A. Heyden, G. Sparr, M. Nielsen et al., Eds., pp. 133-147, Springer, Copenhagen, Denmark, 2002.

[37] J. Puzicha, J. M. Buhmann, Y. Rubner, and C. Tomasi, "Empirical evaluation of dissimilarity measures for color and texture," in Proceedings of the 7th IEEE International Conference on Computer Vision (ICCV'99), pp. 1165-1172, September 1999. 
[38] P. Mitra, C. A. Murthy, and S. K. Pal, "Unsupervised feature selection using feature similarity," IEEE Transactions on Pattern Analysis and Machine Intelligence, vol. 24, no. 3, pp. 301-312, 2002.

[39] P. Cornwell, S. W. Doebling, and C. R. Farrar, "Application of the strain energy damage detection method to plate-like structures," Journal of Sound and Vibration, vol. 224, no. 2, pp. 359-374, 1999.

[40] H. Hu, B.-T. Wang, C.-H. Lee, and J.-S. Su, "Damage detection of surface cracks in composite laminates using modal analysis and strain energy method," Composite Structures, vol. 74, no. 4, pp. 399-405, 2006.

[41] L. H. Yam, Z. Wei, L. Cheng, and W. O. Wong, "Numerical analysis of multi-layer composite plates with internal delamination," Computers and Structures, vol. 82, no. 7-8, pp. 627-637, 2004.

[42] M. Sahin and R. A. Shenoi, "Vibration-based damage identification in beam-like composite laminates by using artificial neural networks," Proceedings of the Institution of Mechanical Engineers C, vol. 217, no. 6, pp. 661-676, 2003. 

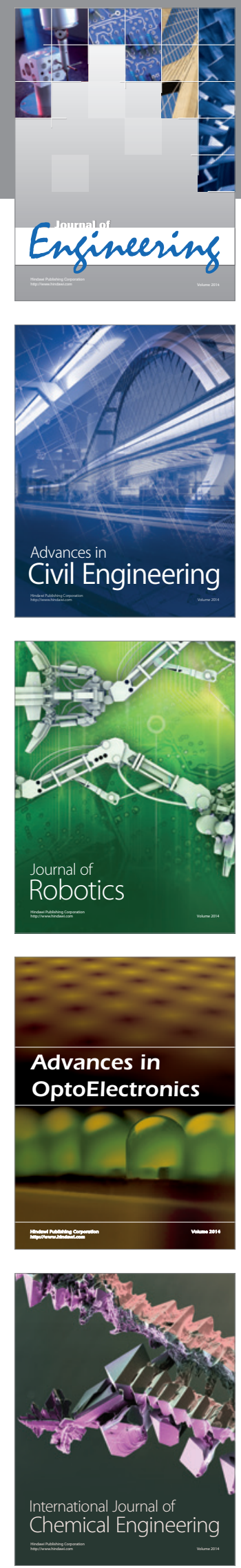

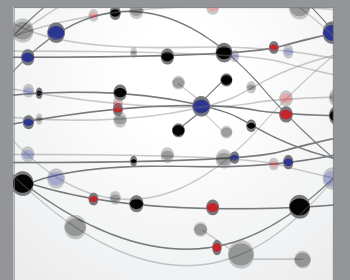

The Scientific World Journal
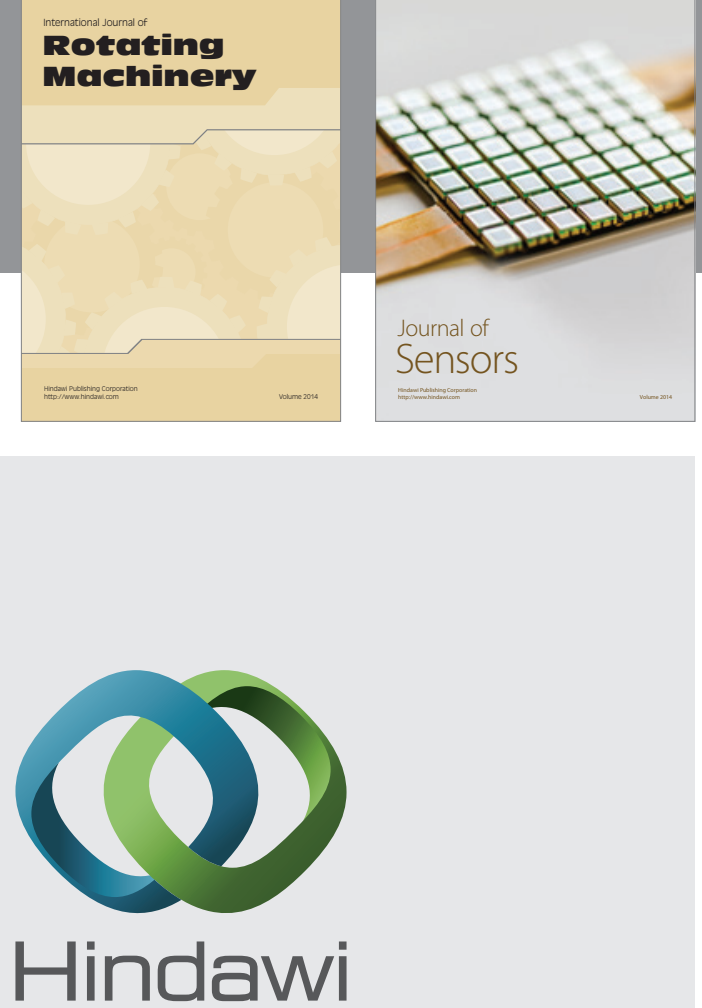

Submit your manuscripts at http://www.hindawi.com
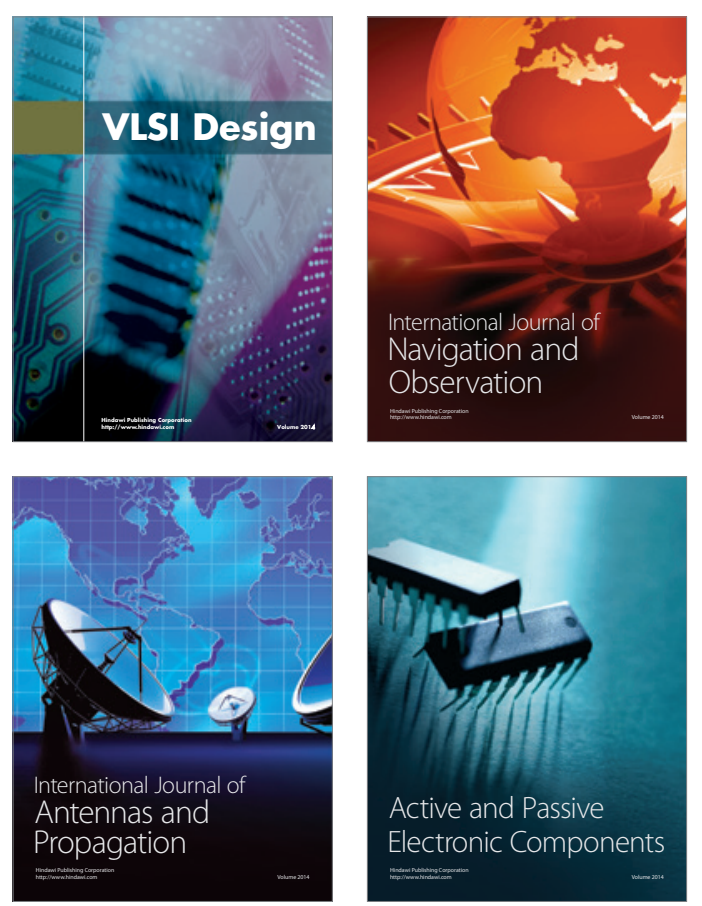
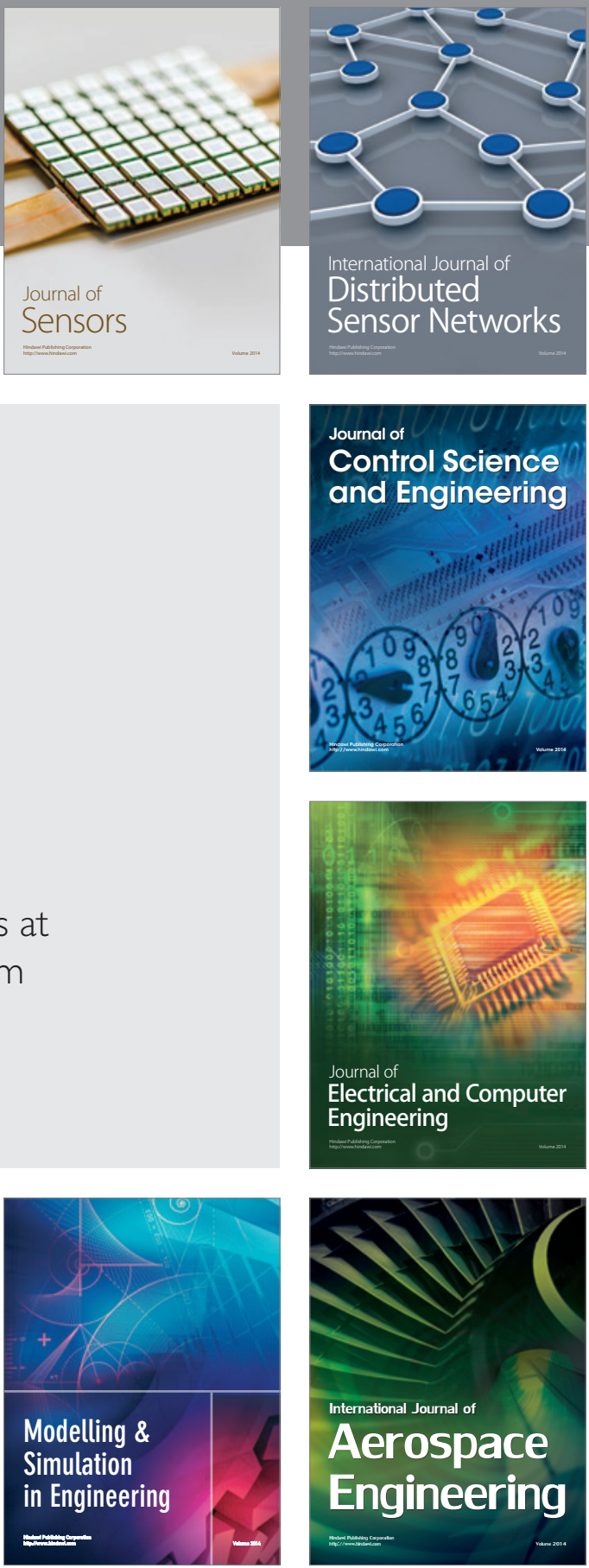

Journal of

Control Science

and Engineering
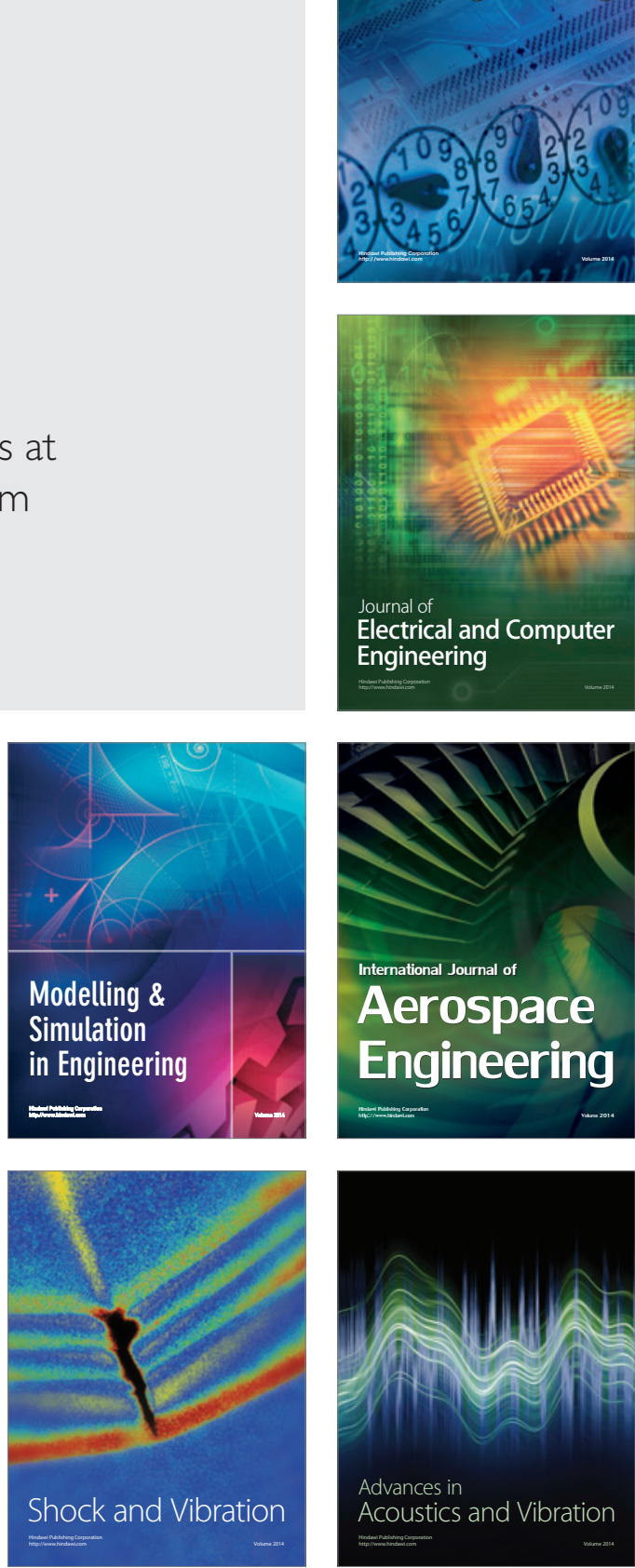